\title{
6 Der „Quakerly approach“ an seinen Grenzen - Das AFSC und Nazi-Deutschland 1933-1939
}

Rufus Jones war sich sicher: Er hatte die Herzen der Gestapo-Männer berührt. In einem 1947 veröffentlichten Artikel für den American Friend blickte er auf eine Reise zurück, die ihn neun Jahre zuvor, wenige Wochen nach den Novemberpogromen in Nazi-Deutschland, bis ins Reichssicherheitshauptamt, die Zentrale der Geheimpolizei, und mithin mitten ins Herz des Terrors geführt hatte. Als Teil einer kleinen AFSC-Delegation war er nach Deutschland gereist, um die deutsche Regierung vom Plan einer Quäkerhilfsaktion zugunsten der verfolgten jüdischen Bevölkerung zu überzeugen. Man sei gekommen, so hieß es in dem Memorandum, das man an Gestapo-Chef Reinhard Heydrich richtete, nicht um zu kritisieren, zu verurteilen oder „to push ourselves in“, sondern um zu „inquire in the most friendly manner whether there is anything we can do to promote life and human welfare and to relieve suffering“. Im Rückblick verstand Jones gut, dass die mündlichen Zusicherungen, die er bei diesem Treffen erhielt, wertlos waren und dass das Treffen im Kern ohne Erfolg geblieben war. Er wusste, was in den folgenden Jahren in Europa geschehen war und welche Rolle die Männer, mit denen er in jenem Dezember gesprochen hatte, dabei gespielt hatten, und er wusste, dass einige seiner Gesprächspartner aus den Reihen der Reichsvereinigung der Juden in Deutschland, darunter die Vorsitzende der Ausreiseabteilung, Cora Berliner, in den Vernichtungslagern ihr Leben verloren hatten. Und dennoch hoffte und glaubte Jones, bei seinen Gestapo-Gesprächspartnern etwas Positives ausgelöst zu haben:

\footnotetext{
I believe for the moment these hard and brutal-minded men, accustomed only to ways of force and violence, found themselves confronted with an unexpected new way of life, which had at its heart another kind of force to which they, in a moment of softness, yielded and paid their respect. If that view is correct the outcome was a miracle wrought by the way of love. The gentleness of the men at the end of our meeting with them, the fact that they went and got our coats and helped us put them on, and shook our hands with good-bye wishes and with a touch of gentleness, made me feel then, and now in retrospect, that something unique had happened in their inside selves. ${ }^{1}$
}

Die Episode spiegelt im Kleinen die Pole wider, welche die Auseinandersetzung des AFSC mit der nationalsozialistischen Diktatur zwischen 1933 und 1939 kennzeichneten: Zwischen störrisch anmutender Naivität und bemerkenswerter Unerschrockenheit, zwischen einem unerschütterlichen Fokus darauf, den Op-

1 Rufus Jones, „Our Day in the German Gestapo“, in: The American Friend, 10/7/1947.

Ә OpenAccess. (C 2022 Daniel Maul, publiziert von De Gruyter. (cc) BY-NC-ND Dieses Werk ist lizenziert unter einer Creative Commons Namensnennung - 4.0 International Lizenz. 
fern des Regimes Hilfe zu leisten, und dem verzweifelten Beharren, selbst im Angesicht unsäglicher Verbrechen an den menschlichen Kern in den Schergen des nationalsozialistischen Verfolgungsapparats appellieren zu können. Wenngleich der Hilfseinsatz zugunsten der Verfolgten des Nazi-Regimes die Grenzen der Politics of Neutrality der Quäker aufzeigte, so hinterließ dieser erste Hilfseinsatz des AFSC seit dem Ende der russischen Hungerhilfe rund zehn Jahre zuvor ein wichtiges Erbe. Die komplexe Natur der Aktion - das Engagement für die Verfolgten in Deutschland zum einen und zum anderen die Bemühungen für die Hunderttausenden, die vor dem Terror der Nazis ins Ausland flohen, griffen ineinander - bedingte die Zusammenarbeit mit einer Vielzahl nationaler und internationaler Institutionen.

Im Wesentlichen ruhte diese Arbeit auf vier Säulen: (1) der amerikanischen Unterstützung für das angloamerikanisch-deutsche Berliner Internationale Quäkerzentrum, (2) der Arbeit für die Flüchtlinge in Paris, Wien und anderen Zentren der Auswanderung, (3) der Mitarbeit im Hochkommissariat des Völkerbundes für die Flüchtlinge aus Deutschland, vor allem in der ersten Phase des Regimes und (4) der Arbeit in den USA selbst, die sowohl praktische Hilfe, vor allem aber auch die Lobbyarbeit zugunsten der Aufweichung der restriktiven Einwanderungsgesetze umfasste. All diese Arbeit fand innerhalb eines großen Netzwerks von Einzelpersonen und Institutionen statt, wobei etwa die Genfer „Quäkerbotschaft“ oder das Berliner Zentrum neue Funktionen übernahmen. Auf diese Weise erweiterte die Arbeit den Blickwinkel und den Aktionsradius des AFSC gerade in einer Phase, in der sich die Friedensbewegung in den USA darauf konzentrierte, den europäischen Ereignissen den Rücken zuzukehren. ${ }^{2}$

\section{Das AFSC und die Machtergreifung}

Schon die Nachrichten von der nationalsozialistischen Machtübernahme im Januar 1933 und von den unmittelbar einsetzenden Verfolgungen in Deutschland lösten im AFSC unmittelbar Reaktionen aus. Am 7. Februar, eine Woche nach Hitlers Vereidigung als Reichskanzler, gab das Komitee seinem concern über die Vorkommnisse in Deutschland Ausdruck. Es wurde beschlossen, die Situation zu beobachten und in enger Abstimmung mit den britischen Quäkern Vorbereitun-

2 Insbesondere zur Arbeit der Quäker in Deutschland nach 1933 ist einschlägig die detailierte Studie von Schmitt, Quakers and Nazis: Inner Light in Outer Darkness; zur Rolle der britischen Quäker siehe den zeitgenössischen Bericht von Lawrence Darton, An Account of the Friends Committee for Refugees and Aliens, First Known as the Germany Emergency Committee of the Society of Friends, 1933-1950 (London: Friends Committee for Refugees and Aliens, 1954). 
gen für mögliche Hilfsaktionen zu treffen. ${ }^{3}$ Dennoch zog das Entsetzen über die Ereignisse in Deutschland im AFSC nicht sogleich auch Handlungen nach sich ganz anders als in Großbritannien: Kaum dass Berichte aus Berlin das Friends House in London erreichten und von „Gewalt- und Terrorakten“ gegen jüdische Einrichtungen sowie gegen Personen sprachen, „well-known to Friends who are victimized for their pacifism, internationalism or socialist sympathies“, ${ }^{4}$ schritt der Friends Service Council (FSC) zur Tat. Bereits Ende März gründete sich ein German Emergency Committee (GEC), das viele Teilnehmer der Hilfsaktion in Deutschland nach dem Ersten Weltkrieg zu seinen Mitgliedern zählte. ${ }^{5}$

Im Vergleich zu Großbritannien liefen die Bemühungen in Philadelphia nur schleppend an. Eine Quäkerdelegation, die im Sommer 1933 nach Deutschland reiste, um die Möglichkeiten einer Hilfsaktion vor Ort zu prüfen, fand zunächst ohne amerikanische Beteiligung statt. Dabei verfolgte das AFSC die deutschen Entwicklungen sehr genau - und dies nicht erst seit Hitlers Machtergreifung. Auf dem Höhepunkt der Weltwirtschaftskrise hatten das AFSC die Hilferufe deutscher Bürgermeister erreicht, die um eine Wiederaufnahme der Kinderspeisungen baten. Wenngleich an eine solche Aktion angesichts der Lage und der Verpflichtungen des AFSC im eigenen Land nicht zu denken war, blieb das Komitee weiterhin Anlaufpunkt für Bitten um Unterstützung von Seiten der deutschen Quäkergemeinde. So war es das AFSC, an das sich die Deutsche Gemeinde wandte, als es galt 1932 ein neues Hauptversammlungshaus im historischen Zentrum des deutschen Quäkertums, im heute niedersächsischen Bad Pyrmont zu erwerben. $^{6}$

Aber auch die politische Entwicklung in Deutschland verfolgten die USQuäker aufmerksam. Bereits 1932 hatte etwa Carolena Wood im AFSC ihren concern angesichts des grassierenden Antisemitismus in Deutschland geäußert. ${ }^{7}$ Die Verbindungen nach Deutschland bestanden zudem über das Berliner Internationale Zentrum weiter und die seit den Jahren der Kinderspeisungen etablierten Kontakte in pazifistische und sozialreformerische Kreise in ganz Deutschland erhielten durch den Machtantritt der Nazis neues Gewicht.

3 AFSC Minutes, 7/2/1933, in: AFSCA, AFSC Minutes 1933.

4 Friends Service Council, Report of Emergency gathering of Friends on the situation in Germany, 27/3/1933, in: LRSF, German Emergency Committee of the Religious Society of Friends in Britain (ohne Signatur)

5 Friends Service Council, Report of Emergency gathering of Friends on the situation in Germany, 27/3/1933, in: LRSF, German Emergency Committee of the Religious Society of Friends in Britain (ohne Signatur).

6 Schmitt, Quakers and Nazis: Inner Light in Outer Darkness, 31-32.

7 AFSC Minutes 24/3/1932, in: AFSCA, AFSC Minutes 1932. 
Exemplarisch war etwa der Fall der Sozialwissenschaftlerin Hertha Kraus, die im Verlauf der 1930er Jahre zu einer der Schlüsselfiguren der Quäkerhilfe zugunsten der Opfer der nationalsozialistischen Verfolgung werden sollte. Kraus war 1920 nach ihrer Promotion in Frankfurt in Berlin mit den Quäkern in Kontakt gekommen (und selbst Quäkerin geworden) und wurde im Anschluss an ihre Tätigkeit bei den Kinderspeisungen zur Geschäftsführerin des Kinderhilfswerks für Ostelbien. Sie gehörte zum Kreis um Friedrich Siegmund-Schultze und Elisabeth Rotten und stand in engem Austausch mit anderen Sozialreformerinnen und Sozialreformern der Weimarer Republik von Gertrud Bäumer über Alice Salomon bis zu Marie-Elisabeth Lüders. Als Sozialdemokratin war Kraus gemeinsam mit Marie Juchatz auch am Aufbau der Arbeiterwohlfahrt beteiligt. 1923 wurde Kraus mit 25 Jahren vom Kölner Oberbürgermeister Konrad Adenauer auf den Posten der Leiterin des städtischen Wohlfahrtsamtes berufen. ${ }^{8}$

Während der gesamten 1920er Jahre blieb Kraus in dieser Position im engen Kontakt mit dem AFSC. In Deutschland unterstützte sie unter anderem auch ein Quäkerhilfswerk für erwerbslose junge Mädchen. Bedeutsamer war, dass sie dem AFSC während der Weimarer Zeit als häufige Referenz für die politische und soziale Lage in Deutschland diente. Die USA hatte sie Ende der 1920er und Anfang der 1930er Jahre wiederholt zu Vortrags- und Studienzwecken bereist und kannte auch viele der Mitglieder des AFSC persönlich. ${ }^{9}$

Als in Prag geborene Jüdin und Sozialdemokratin gehörte Kraus 1933 zu den ersten Opfern der Nazi-Repression nach der Machtergreifung; bereits Anfang April musste sie ihren Kölner Posten aufgeben und floh kurze Zeit später über London ins Exil in die USA. Vor diesem Hintergrund gehörte sie zu den ersten Stimmen, die für eine Hilfsaktion des AFSC zugunsten der Opfer der Nazi-Verfolgung plädierten. Während ihrer letzten Tage in Deutschland zeichnete sie ein düsteres Bild der aufziehenden Diktatur und bat dringend um eine personelle Aufstockung des Berliner Zentrums: „There will be need for friendly visitation, inspection of prisons and concentration camps and possibly personal service in helping some of the victims to go out of the country."10

\footnotetext{
8 Ursula Langkau-Alex, „Hertha Kraus, Die Flüchtlingshilfe der Quäker und die Perzeption von Verfolgten/Geretteten, “ in: Adriane Feustel, Inge Hansen-Schaberg, and Gabriele Knapp (Hg.), Die Vertreibung des Sozialen (München: etk, 2009).

9 So regte Hertha Kraus gegenüber Clarence Pickett eine Zusammenarbeit zwischen Sozialarbeitern aus Deutschland und den USA an. Hertha Kraus an Clarence Pickett 25/8/1932, in: AFSCA, Foreign Service, Country: Germany 1932.

10 Hertha Kraus an Clarence Pickett 27/3/1933, in: AFSCA, General Files, Foreign Service, Country: Germany 1933 (letters to Philadelphia).
} 
Gleichwohl blieb das AFSC zunächst zögerlich. Ende Februar hatte das Komitee die Situation in Deutschland „especially with regard to the treatment of minorities under the new government“ erörtert. Das Protokoll des foreign service committee hielt fest, dass ein „concern on this matter has been placed“ - im AFSCKosmos die formelle Voraussetzung, um Aktionen einleiten zu können. Weiter hieß es dazu: „It was felt that the whole German situation should be discussed by the Service Committee and that any action should be taken in cooperation with English and German Friends. "11 Wie dieser Beitrag der amerikanischen Quäker aussehen sollte, darüber herrschte zunächst jedoch noch Unklarheit. Monate vergingen, in denen das Komitee in einer beobachtenden Haltung verblieb. Im Mai trafen sich Clarence Pickett und Carolena Wood mit Paul Baerwald vom Joint Jewish Distribution Committee, welches das AFSC um die Unterstützung seiner Hilfsaktionen bat. Die Antwort des AFSC war ausweichend. ${ }^{12}$ Im Mai unternahm Gilbert MacMaster dann auf Clarence Picketts Bitte eine ausgedehnte Reise durch Deutschland. Zwei Fragen sollte MacMaster klären: Sollte das AFSC eine Gruppe von „Visiting Friends“ nach Deutschland schicken, die die Moral der deutschen Quäker aufrechterhielt? Und: Waren die Bedingungen gegeben, um eine Hilfsaktion über das Berliner Internationale Zentrum zu initiieren? Nach vier Wochen in Deutschland und einer Vielzahl von Gesprächen mit Vertretern der deutschen Juden und der nationalen Wohlfahrtsverbände, aber auch mit den verstreuten Quäkergemeinden im Land war MacMaster zurückhaltend, was Ersteres betraf, und ausgesprochen skeptisch, was die Erfolgsaussichten einer relief operation anging. Erstens bestehe „no real need“ für Hilfe von außen. Vorerst, so MacMaster, hätten die jüdischen Organisationen genügend Ressourcen. Die Einzelfälle von politisch oder anderweitig Verfolgten, die sich an das Berliner Büro mit der Bitte um Hilfe wandten, könnten hingegen auch ohne eine offizielle Hilfsaktion versorgt werden. Was aus MacMasters Sicht jedoch vor allem gegen eine Hilfsaktion in Deutschland sprach, war, dass diese die Aufmerksamkeit auf die deutschen

11 Minutes Foreign Service Committee 23/2/1933, in: AFSCA, AFSC Minutes 1933.

12 Im Gespräch zwischen Pickett, Richard Cary, Carolena Wood und Paul Baerwald (JDC) ging es bereits um die Frage, ob die Quäker bereit seien, Hilfe an die Juden zu leisten. Pickett ging es auch darum, die jüdische Gemeinde ins Vertrauen zu ziehen und zu erklären, warum sich das AFSC den allgemeinen Protesten gegen die antisemitischen Maßnahmen im Reich nicht anschließen wollte. Die Quäker boten sich nach dem Gespräch Zeit aus, um beurteilen zu können, ob im Quäkersinn unparteiische Hilfe geleistet werden könnte. Clarence Pickett an Carolena Wood 31/03/1933; Clarence Pickett an Gilbert MacMaster 25/5/1933, in: AFSCA, General Files, Foreign Service, Country: Germany 1933 (Gilbert McMaster Correspondence). 
Quäker gerichtet hätte, die ohnedies den Druck der Verfolgung zu spüren begannen. MacMaster plädierte folglich dafür abzuwarten. ${ }^{13}$

So vergingen weitere Monate, bis das AFSC im März 1934 und damit fast ein Jahr nach den britischen Kollegen ein eigenes German Committee einrichtete. Womit ist dieses Zögern zu erklären? Zunächst war der Kontext in den USA ein anderer als in Großbritannien, denn Großbritannien war unmittelbarer mit den Folgen der Nazi-Machtergreifung konfrontiert. Hinzu kam, dass sich in den USA während der ersten Jahreshälfte 1933 ein guter Teil der öffentlichen Aufmerksamkeit auf die Anfänge der New-Deal-Administration Roosevelts und ihr Programm zur Überwindung der Weltwirtschaftskrise konzentrierte. Auch im AFSC, das ja direkt in diesen Neubeginn eingebunden war, richteten sich die meisten Augen auf innere Angelegenheiten. Es war auch, wie gezeigt wurde, keinesfalls entschieden, ob das AFSC in Zukunft überhaupt eine auswärtige Rolle spielen wollte, die über ein allgemeines Interesse und den Unterhalt von „Quäkerbotschaften“ in ausgewählten Ländern Europas hinausging. Und es kam noch ein weiterer Faktor hinzu: Welche Form ein Einsatz in Deutschland unter den gegebenen Bedingungen annehmen könnte, war unklar. Es bestand ja ein deutlicher Unterschied zu allen bisherigen Hilfseinsätzen: Diesmal ging es nicht um die Hilfe für Hungernde, sondern vielmehr um den Schutz von Menschen vor den Maßnahmen einer amtierenden Regierung, die einem solchen Einsatz mit wenig Wohlwollen begegnen würde. Am ehesten war ein solcher Einsatz mit dem Engagement in heimischen industriellen Konflikten vergleichbar, mit dem Unterschied, dass eine der „Konfliktparteien“ in Deutschland nicht nur die Regierung stellte, sondern dass es sich zudem um eine Regierung handelte, deren erklärtes Ziel es war, ihre Gegner sowie Minoritäten auszuschließen, die als Feinde des Volkes ausgemacht worden waren, allen voran die deutschen Juden. ${ }^{14}$

Schließlich war ein Teil der Unsicherheit auf die Schwierigkeiten vieler Quäker zurückzuführen, den Nationalsozialismus als politisch-historisches Phänomen einzuordnen. Selbstverständlich zweifelte niemand, dass es sich um eine gewalttätige Bewegung handelte, die in nahezu all ihren Eigenschaften den Grundüberzeugungen der Quäker entgegenstand. Das Problem aus Sicht vieler Quäker war vielmehr, wie sich der Nationalsozialismus zu der Position verhielt, die Deutschland in der internationalen Ordnung nach Versailles von den Siegern des Weltkriegs zugeteilt worden war. Viele der Führungsfiguren in Philadelphia und London waren in der Kampagne gegen die alliierte Blockade 1919 oder

13 Gilbert MacMaster an Clarence Pickett 29/5/1933, in: AFSCA, General Files, Foreign Service, Country: Germany 1933 (Gilbert McMaster Correspondence).

14 Clarence Pickett an Carolena Wood 31/03/1933, in: AFSCA, General Files, Foreign Service, Country: Germany 1933 (Germany Reports). 
während der Ruhrbesetzung sozialisiert worden oder hatten die Folgen dieser Politik im Rahmen der Kinderspeisungen hautnah miterlebt. Ihr hohes Maß an Identifikation mit ihren deutschen Kontakten ist bereits verdeutlicht worden. Die gesamte Ordnung von Versailles, den Kriegsschuldparagrafen und das folgende Reparationsregime sahen viele von ihnen vor diesem Hintergrund als Ausdruck eines von Rachsucht getriebenen Ungeistes, der die Aussichten auf einen dauerhaften Frieden konterkarierte. Die Schuld für den fehlenden oder zu langsamen Fortschritt bei den internationalen Abrüstungsverhandlungen der 1920er und frühen 1930er Jahre suchten sie - wie viele Pazifisten gerade in England und den USA - bei den Siegern des Krieges; spiegelbildlich erachteten sie auch das deutsche Bestreben, die Ordnung von Versailles zu überwinden, bis zu einem gewissen Grad als legitim. Was 1933 entscheidend war: Es war eben diese Ordnung, die sie dafür verantwortlich machten, Hitler den Boden bereitet zu haben.

Diese Einschätzung erklärt zumindest ein Stück weit die ambivalente Haltung, mit der viele Quäker (auf beiden Seiten des Atlantiks) dem Machtantritt der Nationalsozialisten begegneten: Auf der einen Seite stand der Schock und die Abscheu gegenüber den massiven und brutalen Verfolgungen. Auf der anderen Seite wollten viele das neue Regime nicht ausschließlich anhand seiner Exzesse bewerten. Insbesondere galt es aus Sicht vieler zu vermeiden, mit Wort oder Tat zum wachsenden anti-deutschen Gefühl zu Hause beizutragen. Bei einer der ersten Sitzungen des deutschen Notfallkomitees in London im März 1933 appellierte Hertha Kraus selbst, die gerade ihren Verfolgern nach Großbritannien entkommen war, die nun notwendige Hilfe für die Verfolgten mit der „right publicity“ zu flankieren, um zu vermeiden, dass diese Aktionen in ,indiscriminating AntiGerman feeling“ mündeten. ${ }^{15}$ Andere gingen weiter und meinten, konstruktive und sogar vielversprechende Elemente in dem neuen Regime zu sehen, während sie die Verfolgungen als Auswüchse einer außerordentlichen, revolutionären Situation interpretierten. Für Walter Kotschnig, einen Unternehmer aus Philadelphia, der Anfang 1933 im inoffiziellen Auftrag des AFSC eine Informationsreise nach Deutschland unternahm, war der richtige Umgang mit dem NS-Regime „not simply an attitude of condemnation and criticism“, sondern ein „constructive approach to what is at present the problem of Europe“. Für Kotschnig war die nationalsozialistische Herrschaft sowohl ein Ergebnis internationaler Faktoren Deutschland als Opfer der Post-Versailler Ordnung und der Heuchelei der Sieger -

15 Friends Service Council, Report of Emergency gathering of Friends on the situation in Germany, 27/3/1933, in: LRSF, German Emergency Committee of the Religious Society of Friends in Britain (ohne Signatur). 
als auch interner Faktoren, etwa der Weimarer Verfassung, die nicht zu einer nach seiner Einschätzung grundsätzlich autoritär gesinnten Gesellschaft passte. ${ }^{16}$

Ähnlich ambivalent war die Haltung von Douglas Steere, einem weiteren AFSC-Mitarbeiter des Berliner Quäkerzentrums. Seine Sorge galt (aus anderen Gründen als bei MacMaster) den deutschen Quäkern, die das NS-Regime im Lichte der Verfolgungen als den leibhaftigen Anti-Christen betrachteten, da er ihre Haltung, bei allem Verständnis, nicht im Einklang mit einem „quakerly approach“ sah, der sich stets darauf konzentrieren müsse, was am Gegenüber „konstruktiv“ war. Für ihn war der richtige Umgang mit dem Nationalsozialismus vergleichbar mit jenem eines Kriegsdienstverweigerers mit dem Staat, der ihn wegen seiner Überzeugungen verfolgte und inhaftierte: „in no sense condemning the State or hating it for the treatment it gave him, but instead holding the state to his ,true self' and upholding the hope that there would be something, in the minds of those who adminster the punishment (...) that will ultimately respond to our testimony“ ${ }^{17}{ }^{17}$ Auch andere Vertreter des AFSC hielten es sowohl für wünschenswert als auch für möglich, dass Quäker den Widerspruch überbrücken könnten, der zwischen ihrer Arbeit für die Opfer der nationalsozialistischen Unterdrückung und dem Anspruch klaffte, Teil eines größeren allumfassenden Friedens- und Versöhnungswerks zu sein. ${ }^{18}$ Solche Stimmen blieben erwartungsgemäß nicht ohne Widerspruch. Henry Cadbury etwa erregte 1934 einiges Aufsehen und Unverständnis, als er von der New York Times mit der Aussage zitiert wurde, dass „Good will, not hate or reprisals will end, or offset the evils of Hitler's government's persecution of Jews“. Empörte Reaktionen der jüdischen Gemeinde, aber auch aus Kreisen der Friedensbewegung in den USA waren die Folge. ${ }^{19}$

Als Clarence Pickett Anfang 1934 Deutschland besuchte, waren viele Hoffnungen auf eine Normalisierung nach den revolutionären Anfängen bereits verflogen. Was Pickett in Deutschland sah, war die „rule of the roughnecks“. Und dennoch wollte auch Pickett „certain great motives at work“ erkennen - „blindly to be sure - which we must recognize“. Allerdings stellte Pickett fest, dass die

16 „We can see it here in Geneva, where all those forces who have blocked real disarmament up to now and who to a large extent are responsible for what is happening in Germany are now proclaiming that because of the German attitude all disarmament is utterly impossible." Walter Kotschnig an Clarence Pickett 15/5/1933, in: AFSCA, General Files, Foreign Service, Country: Germany 1933 (Letters to Philadelphia, Letters to Germany).

17 Memo Douglas Steere für Clarence Pickett 10/10/1933, in: AFSCA, General Files, Foreign Service, Country: Germany 1933 (German Yearly Meeting).

18 Gilbert MacMaster an Bertha Bracey (FSC London) 27/10/1933, in: LRSF, German Emergency Committee, Berlin Center (ohne Signatur).

19 Good-Will barred to Nazis by Rabbis; Wise leads wave of objection to advice by Cadbury of Society of Friends, in: New York Times, 16/6/1934. 
Erfolge, mit denen sich die Nazis brüsteten, in erster Linie auf dem Rücken bestimmter Teile der Bevölkerung errungen waren, insbesondere auf dem der Juden und auch von Frauen. Wenn Deutschland eine „menace to World Peace“ war, wovon Pickett durchaus überzeugt war, dann ,not primarily because she plans a war; but because she is implanting on a wide scale the conviction that force is the only way for Germany to get her former recognition“. Und dennoch: Auch Pickett konnte nicht umhin festzustellen, dass es der Versailler Vertrag und die Behandlung Deutschlands durch die Sieger seit dem Ende des Krieges waren, die nun ,all are bringing their tragic harvest“. ${ }^{20}$

\section{Im Auge des Terrors. Das Berliner Internationale Zentrum 1933-1935}

Ein wesentlicher Teil der Arbeit des AFSC für Deutschland in den ersten Jahren nach der Machtergreifung konzentrierte sich auf die Arbeit des Berliner International Center, der größten unter allen „Quäkerbotschaften“ in Europa. ${ }^{21}$ Eine der ersten unmittelbar ergriffenen Maßnahmen bestand deshalb in der Aufstockung der finanziellen Mittel für die Arbeit in Berlin. Anfang 1933 lag die Leitung in der Hand von Richard Cary, einem Journalisten aus Baltimore und Veteranen der Kinderspeisungen der 1920er Jahre. Als Cary Ende November 1933 völlig unerwartet im Alter von nur 32 Jahren verstarb, übernahm sein britischer Kollege Thomas „Corder“ Catchpool die Führung. Carys Position als Vertreter des AFSC im Zentrum übernahm Gilbert MacMaster, der freilich nur bedingt ein vollwertiger Ersatz für seinen Vorgänger sein konnte, da er seinen Wohnsitz in Basel beibehielt und zwischen der Schweiz und Berlin pendelte.

MacMasters sporadische Anwesenheit machte Catchpool automatisch zur zentralen Figur im Umgang des Zentrums mit den neuen nationalsozialistischen Machthabern und gab seiner Haltung großes Gewicht. Catchpool war dabei zuallererst ein Mann von tiefem Glauben und Überzeugungen, für die er bereit war, ein hohes persönliches Risiko einzugehen: Als Kriegsdienstverweigerer (der auch den Dienst in einer der Quäkerhilfseinheiten aus prinzipiellen Gründen abgelehnt hatte) hatte er den längsten Teil des Ersten Weltkriegs im Gefängnis zugebracht. Danach war er in der Fight-the-Famine-Bewegung aktiv gewesen und hatte für längere Zeit an den Studenten- und Kinderspeisungsaktionen in Deutschland

20 Clarence Pickett Memorandum on trip to Germany 6/5/1934, in: AFSCA, General Files, Foreign Service, Country: Germany 1934.

21 Die Arbeit des Berliner Zentrums dokumentiert ausführlich Schmitt, Quakers and Nazis: Inner Light in Outer Darkness., 54-74; 111-121. 
teilgenommen. Seitdem unterhielt er enge Kontakte ins deutsche pazifistische und sozialreformerische Milieu. Nach zehn Jahren in einer lebensreformerischen Landkolonie in England war er 1931 mit seiner Familie nach Berlin zurückgekehrt und hatte diese Verbindungen reaktiviert. ${ }^{22}$ Bei Machtantritt der Nationalsozialisten galt Catchpools Aufmerksamkeit in erster Linie den Opfern der nun einsetzenden Verfolgung, von denen er viele persönlich kannte und zu seinen Vertrauten zählte. Als es in den ersten Monaten des Terrors vielen Verfolgten mit Geld, Lebensmitteln oder Kleidung aushalf und in einigen Fällen auch Unterschlupf oder Hilfe bei der Flucht aus Deutschland gewährte, geriet Catchpool unmittelbar ins Visier der neuen Machthaber. ${ }^{23}$

Im April 1933, nachdem sich die Quäker gegen die von den Nazis orchestrierten Boykotte jüdischer Geschäfte und Institutionen ausgesprochen hatten, wurde er zum ersten Mal von der Gestapo zum Verhör vorgeladen. Ihm wurden „Greuelberichte“ und Kontakte zu Gegnern des Regimes vorgeworfen. Nach drei Tagen in Haft wieder auf freien Fuß gesetzt, blieb gleichwohl sein Pass für weitere 18 Monate eingezogen; die Anklage gegen ihn wurde formal nie fallengelassen. ${ }^{24}$ Trotzdem setzte er die Arbeit unbeirrt fort und das Zentrum diente auch in der Folge als Anlaufstelle für Verfolgte.

Eine klare Regel, welche Personengruppen in die Verantwortung der Quäker fallen sollten, gab es nicht; ab Ende 1933 bestand aber eine informelle Arbeitsteilung mit jüdischen Hilfsorganisationen, wobei sich die Quäker im Wesentlichen auf nichtjüdische Verfolgte sowie die Gruppe der sogenannten nichtarischen Christen konzentrierten, das heißt auf Personen, die als Christen geboren worden oder die zum Christentum konvertiert waren, aber vom NS-Staat aufgrund ihrer Herkunft als „Halb-“ oder „Vierteljuden“ bezeichnet wurden. Im Oktober 1933 half das Berliner Zentrum, ein Erholungsheim für Personen zu eröffnen, die in KZs oder Gefängnissen eingesperrt gewesen waren. Als Lokalität diente zunächst ein

22 Eine zeitlich nicht verortbare Biografie auf Deutsch, verfasst von Otto Peetz findet sich in den Corder Catchpool Papers, die Teil der Swarthmore Peace Collection sind. Die bislang einzige Biografie hat Catchpools Mitstreiter im FSC, William Hughes, verfasst: William Ravenscroft Hughes and Thomas Corder Pettifor Catchpool, Indomitable Friend. The Life of Corder Catchpool, 18831952. [with Portraits.] (London: Housmans, 1956).

23 Die Bandbreite der Menschen, die im Berliner Zentrum Zuflucht suchten, wurde wie folgt beschrieben: „Social Democrats or Socialists, people who had aided or spoken out for Jews, who had been members of the World League of Youth for Peace or other pacifist internationalist institutions, people who held generally ,liberal views on racial and international questions', who had attended Friendship Camps in England“, Bericht n.n. für das GEC, 27/3/1933, in: 27/3/1933, in: LRSF, German Emergency Committee of the Religious Society of Friends in Britain (ohne Signatur). 24 Die Anzeige gegen Catchpool durch den SS-Mann Otto Konold 30/3/1933, in: AFSCA, General Files, Foreign Service, Country: Germany 1933 (Corder Catchpool's Journals from Berlin). 
Hotel in Falkenstein im Taunus, später wurde dafür das Tagungshaus der deutschen Quäker in Bad Pyrmont genutzt. ${ }^{25}$

Während ihm diese Aktivitäten hohes Ansehen verschafften, haftete Catchpool gleichzeitig der zweifelhafte Ruf an, seine eigene „personal policy of Nazi appeasement“ zu verfolgen. ${ }^{26}$ Wie viele andere Pazifisten seiner Generation teilte auch Catchpool die entschiedene Ablehnung der Versailler Ordnung. Wie keine zweite unter den führenden Quäkerpersönlichkeiten der Zeit repräsentierte er darüber hinaus ambivalente Haltung vieler Friends zum Nationalsozialismus. Als Mitglied der Labour Party und nach eigener Beschreibung „christlicher Sozialist“ und Demokrat über den Verdacht erhaben, übertriebene Sympathien für die NaziBewegung zu hegen, gehörte er gleichfalls entschieden ins Lager jener, die Hitler als Konsequenz der alliierten Politik seit Versailles betrachteten, die der jungen Weimarer Demokratie die Grundlagen entzogen habe. Seine gesamte Einschätzung der Lage 1933 und in den darauffolgenden Jahren ging von dieser Grundüberzeugung aus.

Mitte 1933 schickte er einen Bericht mit dem Titel „Reflections: on our attitude towards the National-Socialist revolution in Germany“ an den FSC in London, in dem er „in quakerly tradition“ dafür plädierte, offen gegenüber dem neuen Regime zu sein, Gemeinsamkeiten zu suchen und unter den neuen Machthabern jene $\mathrm{zu}$ identifizieren, die für eine Zusammenarbeit $\mathrm{zu}$ gewinnen waren. Seine Prämisse war, dass Frieden das ultimative Ziel des NS-Regimes sei und dass deshalb bestehende Meinungsverschiedenheiten durch einen Dialog zwischen Menschen guten Willens auf beiden Seiten ausgeglichen werden könnten. Catchpool selbst ließ diesen Worten immer wieder Taten folgen und nahm Kontakt in Regierungskreise sowie zu dem Regime nahestehenden Persönlichkeiten auf. Zum Beweis, dass die Quäker gegenüber der Nazibewegung nicht voreingenommen seien, nahm Catchpool auch an Hilfsaktionen für inhaftierte Nazi-Aktivisten außerhalb Deutschlands wie etwa in Memel und in Danzig teil. In späteren Jahren folgten weitere Beispiele dessen, was Catchpool im Quäkersinn als „pacifist diplomacy“ verstand: Er unterstützte eine Kinderspeisung im deutschsprachigen Sudetengebiet der Tschechoslowakei und ergriff Partei für die deutsche Seite im polnisch-deutschen Grenzkonflikt in Oberschlesien. ${ }^{27}$

25 Beide Einrichtungen wurden bis 1939 von ca. 800 Personen genutzt. Darton, An Account of the Friends Committee for Refugees and Aliens, First Known as the Germany Emergency Committee of the Society of Friends, 1933-1950, 7-8. Die Geschichte des Rest Home findet sich auch in Claus Bernet, „Das Rest für Home Verfolgte des Dritten Reiches,“ Exil. Forschung - Erkenntnisse - Ergebnisse 2 (2004), $75-81$.

26 Schmitt, Quakers and Nazis: Inner Light in Outer Darkness, 66.

27 Schmitt, Quakers and Nazis: Inner Light in Outer Darkness, 89-92. 
Bei all dem agierte Catchpool als Teil eines breiteren Netzwerks britischer Pazifisten, hauptsächlich mit sozialistischem und liberalem Hintergrund, darunter Lord Noel-Buxton, George Lansbury und Lord Hurtington. Ihre Zusammenarbeit beruhte auf dem Verständnis, dass der Frieden erhalten werden könnte, wenn die legitimen Forderungen Deutschlands bezüglich der Beseitigung der Versailler Ordnung erfüllt würden. Diese Gruppe, die bei verschiedenen Gelegenheiten zu Gesprächen mit hohen Vertretern des Nazi-Regimes geladen wurde, darunter etwa Heinrich Himmler, traf sich regelmäßig in einem englischdeutschen Kreis, dem von deutscher Seite der Leiter der Deutschen Akademie für Auswärtige Politik, Fritz Berber, angehörte. Catchpool gehörte über den gesamten Zeitraum ihrer Existenz zum harten Kern dieser Gruppe, und hielt die Kontakte zu Berber selbst dann noch am Leben, als das Scheitern des Münchner Abkommens die Politik des appeasement für die meisten Beteiligten endgültig diskreditiert hatte. ${ }^{28}$

Unter Quäkern in England und den USA und insbesondere im Kreis derjenigen, die sich mit der deutschen Situation direkt beschäftigten, lösten die Aktivitäten Corder Catchpools vom ersten Moment an geteilte Reaktionen aus. So griffen Carl Heath, Joan Fry und einige andere führende britische Quäker Catchpool heftig an, kritisierten ihn als „convert to Nazism“ und „pro-Nazi“ und stellten wiederholt sein Recht in Frage, für die Society of Friends zu sprechen. Andere wie Heaths Nachfolger als Vorsitzender des FSC, Paul Sturge, waren hingegen offener für Catchpools Kurs. Auch im AFSC war man hinsichtlich Catchpools Ansatz zwiegespalten. Einige mochten diesen für „naiv“ und eigensinnig halten. Im Wesentlichen jedoch teilten prominente Vertreter des AFSC zumindest anfänglich Catchpools Haltung, die Opfer in den Vordergrund zu stellen, gleichzeitig jedoch dem Regime mit einem unvoreingenommenen „quakerly approach“ zu begegnen. Richard Cary hatte während der ersten Monate des Regimes eine ähnliche Linie verfolgt: Auch er wollte zunächst an das konstruktive Potenzial des Nationalsozialismus glauben und war bereit, dessen Gewaltexzesse als Kinderkrankheiten $\mathrm{zu}$ entschuldigen. In seinem ersten Bericht für das AFSC nach der Machtergreifung wandte er sich recht deutlich gegen Stimmen wie die von Hertha Kraus, die für eine klarere Abgrenzung plädierten. ${ }^{29}$

Auch sein Nachfolger Gilbert MacMaster folgte zunächst dieser Linie. Auf den Posten in Berlin war er dank seiner Erfahrungen in Berlin, vor allem aber aufgrund seiner vielen Kontakte nach Deutschland berufen worden. Bereits während der

28 Zu Berber siehe Katharina Rietzler, „Counter-Imperial Orientalism: Friedrich Berber and the Politics of International Law in Germany and India, 1920s-1960s, “ Journal of Global History 11, 1 (2016).

29 Schmitt, Quakers and Nazis: Inner Light in Outer Darkness, 63-64. 
1920er Jahre hatte MacMaster sein besonderes Interesse für Minoritätenfragen entwickelt, das er auch nach 1933 beibehielt. ${ }^{30}$ Auch in die jüdische Gemeinde pflegte er seit den 1920er Jahren enge Beziehungen. Sein Bekanntenkreis umfasste zur selben Zeit jedoch auch Personen, die der Nazibewegung nahestanden, darunter Ernst Hanfstängel, einer der Vertrauten Hitlers aus der „Bewegungszeit“, oder der Oberstaatssekretär in der Reichskanzlei und spätere Geschäftsträger ${ }^{31}$ der deutschen Botschaft in Washington von 1938 bis 1941, Hans Thomsen. MacMaster verwendete diese Kontakte wie Catchpool vor allem dafür, um auf individueller Ebene Erleichterungen für Persönlichkeiten zu erreichen, die mit den Quäkern in Verbindung standen und deshalb ins Visier des Regimes geraten waren. Zwischen 1933 und 1935 besuchte er regelmäßig Konzentrationslager und wurde zugunsten einiger hochrangiger Verfolgter tätig, wie etwa der SPD-Politiker Gerhart Seger, Ernst Heilmann, Ernst Reuter und Rudolf Küstermeier (des späteren ersten Chefredakteurs der Welt), des Rechtsanwalts Hans Litten sowie, als prominentester Fall, des Publizisten Carl von Ossietzky. ${ }^{32}$

Aus der Korrespondenz MacMasters geht hervor, wie tief gespalten er mit Blick auf diese Aktivitäten in Deutschland war. So zweifelte er mit zunehmender Dauer am Sinn der Lobbyarbeit für die Verfolgten. In einigen Fällen, so vermerkte er kritisch in einem Schreiben an Clarence Pickett, mochte der Einsatz der Quäker sogar dazu geführt haben, dass sich die Aussichten der Inhaftierten verschlechtert hatten. ${ }^{33}$ Gerade deshalb erwog er, ob es nicht besser sei, „to associate more with the ones who could prevent many of the troubles and the suffering of the world and not always with the victims. I personally feel more and more the desire to get to the man who is responsible for the welfare or the suffering of a thousand people than to become so busied with thousands that I have no time for. “34

30 Seine Aktivitäten erstreckten sich vor allem auf die deutsch-polnischen Beziehungen, aber in den 1930er Jahren engagierte MacMaster sich auch im Rahmen der Frage der deutschsprachigen Südtiroler, die nach 1919 zu Italien gekommen waren und seit Mussolinis Machtantritt einer rigiden „Entnationalisierungspolitik“ unterlagen. MacMasters Aktivitäten werden durch seine umfangreiche Korrespondenz mit Clarence Pickett insbesondere in den Jahren 1933 bis 1935 greifbar, siehe den Ordner „Letters Gilbert MacMaster/Correspondence Gilbert MacMaster“ in: AFSCA, General Files, Foreign Service, Country: Germany 1933-1935.

31 Thomsen leitete die Botschaft nach der Abberufung des Botschafters Hans Dieckhoff von 1938 bis zum Kriegseintritt der USA im Dezember 1941 geschäftsführend.

32 Die Kampagne zur Freilassung von Ossietzky begleiteten MacMaster und andere Quäker aktiv bis zu Ossietzkys Tod im Jahr 1938.

33 Gilbert MacMaster an Clarence Pickett 10/11/1934, in: AFSCA, General Files, Foreign Service, Country: Germany 1934 (Gilbert MacMaster Correspondence).

34 Gilbert MacMaster an Clarence Pickett 10/11/1934, in: AFSCA, General Files, Foreign Service, Country: Germany 1934 (Gilbert MacMaster Correspondence). Nur wenige Tage später schrieb er 
Das vielleicht beste Beispiel für das Beharren auf einer „quäkerlichen“ Haltung gegenüber dem Nationalsozialismus, trotz oder gerade wegen der Verfolgungen, ist eine Episode aus dem Jahr 1934. Nach dem gescheiterten Putschversuch österreichischer Nationalsozialisten gegen das Ständeregime von Kanzler Engelbert Dollfuß im Juli dieses Jahres trat eine Organisation mit dem Namen „Brüder in Not“ an die Quäker in Berlin mit dem Vorschlag heran, den Familien der nach dem Putsch inhaftierten Nationalsozialisten Hilfe zu leisten. Brüder in Not war eine Vereinigung, die, geleitet von dem deutschen Mennoniten Benjamin Heinrich Unruh, Hilfe für die deutschen Minderheiten insbesondere in Osteuropa leistete; die Mitgliedschaft reichte von der katholischen Caritas über verschiedene Deutschtumsvereine bis zum NS-Wohlfahrtsverband. Im Kern handelte es sich allerdings unverkennbar um eine Vorfeldorganisation der gleichgeschalteten nationalsozialistischen Deutschtumspolitik, über deren Intentionen sich niemand Illusionen machen konnte: „Brüder in Not“ wollte die Quäker als politisch unverdächtigen Kanal für ihre Hilfsgelder nutzen. ${ }^{35}$ Anfänglich waren auf Quäkerseite alle Beteiligten offen für eine solche Zusammenarbeit. Clarence Pickett und Gilbert MacMaster kannten Unruh noch von einer Aktion am Rande von Picketts Europareise 1930, als der AFSC-Generalsekretär sich auf Bitte Unruhs für die in der Sowjetunion verfolgten Mennoniten verwendet hatte. ${ }^{36}$

Für Pickett und MacMaster schien sich anfänglich mit der Brüder in Not-Initiative in Österreich eine Gelegenheit zu ergeben, dem Nazi-Regime die Unparteilichkeit der Quäker zu zeigen - insbesondere vor dem Hintergrund, dass die Quäkerbotschaft in Wien unter der Leitung Emma Cadburys nach der Niederschlagung des österreichischen Arbeiteraufstands im Frühjahr desselben Jahres auch den meist sozialistischen Opfern Hilfe geleistet hatte. ${ }^{37}$ Am Ende, nach über einem halben Jahr diskreter Verhandlungen, zog sich das AFSC von der Sache zurück, nachdem Pickett und MacMaster zu dem Schluss gelangt waren, dass eine

Pickett einen weiteren Brief, der eine weitaus optimistischere Note enthielt. Gerade hinsichtlich ihrer Unvoreingenommenheit erfahre er viel Zuspruch, berichtete er: „There is widespread growing faith in what Friends undertake is a most remarkable thing. We as American Friends must just not allow the depression or anything else to shut us out of our share in this." Gilbert MacMaster an Clarence Pickett 14/11/1934, in: AFSCA, General Files, Foreign Service, Country: Germany 1934 (Gilbert MacMaster Correspondence).

35 Aus der Korrespondenz MacMasters mit anderen geht hervor, dass sich alle Beteiligten von Anfang an der politischen Brisanz bewusst waren. Gilbert MacMaster an Fred Tritton 28/8/1934; Gilbert MacMaster an Clarence Pickett 31/8/1934, in: AFSCA, General Files, Foreign Service, Country: Germany 1934 (Gilbert MacMaster Correspondence).

36 Siehe Kapitel 5.

37 Gilbert MacMaster an Clarence Pickett_2/10/1934, in:_AFSCA, General Files, Foreign Service, Country: Germany 1934 (Gilbert MacMaster Correspondence). 
offizielle Aktion zugunsten der inhaftierten österreichischen Nationalsozialisten als Parteinahme des AFSC aufgefasst worden wäre. ${ }^{38}$ Im September bezeichnete Pickett gegenüber Henry Cadbury das Brüder in Not-Angebot als nicht ,active“, war jedoch weiter bereit, nach Wegen zu suchen, als Geste des guten Willens der Quäker etwas für die „Nazi sufferers in Austria“ zu tun. ${ }^{39}$ Erst die Verkündung der sogenannten Nürnberger Rassengesetze wenige Tage später brachte ein Umdenken in Gang, so dass es zu keinen weiteren ähnlichen Initiativen mehr kam.

Wenn Vertreter des AFSC zur selben Zeit zunehmend auf Distanz zu Catchpool gingen, so geschah dies wohl nicht, weil sie seinen Kurs im Umgang mit dem Nationalsozialismus grundsätzlich ablehnten. In der „Brüder in Not“-Initiative waren beide auf derselben Wellenlänge. Für wachsende Spannungen waren eher zwei andere Faktoren entscheidend, die gleichwohl mit dieser Strategie in Zusammenhang standen. Einerseits trieben das AFSC und MacMaster die Sorge um die deutschen Quäker um, denn für diese stellte die Art, wie das Berliner Zentrum seine Hilfsarbeit für die Verfolgten betrieb, eine permanente Gefahr dar.

Dies galt insbesondere in den Fällen, in denen sich die Quäker für exponierte Regimegegner wie von Ossietzky oder Küstermeier einsetzten. Während die Briten und Amerikaner im Zentrum auf den Schutz ihrer jeweiligen diplomatischen Vertretungen zählen konnten, warfen gerade solche Aktionen ein Schlaglicht auf die kleine Gruppe der deutschen Quäker, deren Lage sich seit Clarence Picketts Besuch in Deutschland 1933 ebenfalls dramatisch verschärft hatte. Für diese stellte das Zentrum einerseits eine wichtige Verbindungslinie zu den großen Gemeinden in Großbritannien und den USA dar, die ihnen unter Umständen Schutz bieten konnte. Andererseits barg gerade der internationale Charakter des Zentrums ein großes Risiko. Für Hans Albrecht, einen Hamburger Schiffsinspektor, der als Leiter des Deutschen Jahrestreffens fungierte, des höchsten Gremiums der deutschen Quäker, bestand die Herausforderung darin, die kleine deutsche Gemeinde zu schützen. Dafür hielt er einerseits die auswärtigen Kontakte so lange wie möglich aufrecht und versuchte gleichzeitig, alles $\mathrm{zu}$ vermeiden, was die Aufmerksamkeit der Nazis erweckt hätte. Für viele deutsche Quäker war das Leben im Nationalsozialismus ohnedies voller Gefahren und Bedrängnisse. Nicht wenige bezahlten ihren Pazifismus oder die bloße Gemeindezugehörigkeit mit

38 Aus einem Brief MacMasters an Pickett geht hervor, dass das AFSC unter der Prämisse bereit war, Hilfe zu leisten, dass die Quäker dabei nicht offiziell in Erscheinung traten. Diese Bedingung war für Brüder in Not nicht akzeptabel, so dass die Sache versandete. Gilbert MacMaster an Clarence Pickett 13/2/1935, in: AFSCA, General Files, Foreign Service, Country: Germany 1935 (Gilbert MacMaster Correspondence).

39 Clarence Pickett an Henry Cadbury 18/9/1935, in AFSCA, General Files, Foreign Service, Country: Germany 1935 (Germany General). 
Gefängnis und Konzentrationslager; andere kehrten den Quäkern den Rücken oder verließen das Land. ${ }^{40}$ Beim AFSC und seinen Vertretern in Deutschland konnten die deutschen Quäker dabei auf mehr Verständnis für ihre Nöte hoffen als bei den britischen Glaubensgenossen. Dies lag vor allem daran, dass es in Deutschland die US-Quäker waren, die im Gefolge der Kinderspeisungsaktionen eine Art inoffizielle Patenschaft für die deutsche Gemeinde übernommen hatten. ${ }^{41}$

Zum Problem für die deutschen Quäker wurde neben Catchpools Hilfe für die Verfolgten denn auch die Paralleldiplomatie, die er und einige weitere britische Quäker zur selben Zeit in Deutschland betrieben. ${ }^{42}$ Dabei versuchten Catchpool und der FSC-Vorsitzende Paul Sturge, Aktionen wie in Schlesien oder im Kontext der „Brüder in Not“-Initiative als Belege des guten Willens und der neutralen Position der Quäker einzusetzen. So etwa, als beide 1935 gemeinsam einen an Hitler direkt gerichteten Brief entwarfen. Die Quäker, so hieß es darin, seien „dem deutschen Volke seit vielen Jahren in wahrhafter Freundschaft zugetan“, wobei eine Linie von der Hilfe für die deutschen Zivilinternierten im Ersten Weltkrieg über die Kinder- und Studentenspeisungen während der Ruhrbesetzung und schließlich in die Gegenwart gezogen wurde. Man sehe „mit Kummer die Wirkung auf die freundschaftliche Meinung im Ausland, besonders in England, hervorgerufen durch die fortdauernde Gefangenhaltung in $\mathrm{KZ}$ und Gefängnissen von Zivilisten ohne gerichtliches Verfahren, unter außerordentlich harten Bedingungen“. Es sei folglich „lediglich Deutschlands guter Ruf in England“, der den Quäkern am Herzen liege. Der Brief bat um eine Generalamnestie für sämtliche politischen Gefangenen im Reich, eine Geste, die wie keine zweite „wirksam die Achtung für Deutschland im Auslande festigen“ würde. ${ }^{43}$ Um diesen Brief übergeben zu können, bemühte sich Catchpool sogar um eine persönliche Audienz bei Hitler. $^{44}$

40 Schmitt, Quakers and Nazis: Inner Light in Outer Darkness, 42-53; 122-149.

41 Memo Douglas Steere für Clarence Pickett 10/10/1933, in: AFSCA, General Files, Foreign Service, Country: Germany 1933 (German Yearly Meeting).

42 Gilbert MacMaster an Clarence Pickett 14/1/1935, in AFSCA, General Files, Foreign Service, Country: Germany 1935 (Gilbert MacMaster Correspondence).

43 Brief-Entwurf Paul Sturge an Adolf Hitler (Anfang 1935), LSRF, German Emergency Committee 1935 (ohne Signatur).

44 Aus den Akten geht hervor, dass Catchpool große Hoffnungen in eine Audienz bei Hitler selbst setzte; auch bekunden sie die rege direkte Diplomatie, die er zugunsten der politischen Gefangenen betrieb. Seinen Gesprächspartner Hans Thomsen in der Reichskanzlei erinnerte er an die Kinderspeisungen und die Hilfe während der französischen Okkupation der Ruhr. Wäre es tatsächlich zu einem Gespräch mit Hitler gekommen, hätten neben Catchpool Gilbert MacMaster und William Hughes die Quäker vertreten. Gespräch mit Dr. Thomsen (Reichskanzlei) 6/3/1935, in: 
Während solche Initiativen erfolglos verpufften, erwiesen sich die Aktivitäten von William Hughes, der seit 1933 als eine Art geheimer Emissär des britischen GEC Deutschland bereiste, als folgenreicher. Hughes nahm einerseits wiederholt Einladungen des Regimes zu Besuchen etwa im Konzentrationslager Dachau an, auch um Informationen über bestimmte Insassen an die Angehörigen weitergeben $\mathrm{zu}$ können. ${ }^{45}$ Als heikler erwies sich, dass Hughes zeitgleich über Untergrundkanäle auch Hilfsmittel des britischen Emergency Committee an Verfolgte verteilte und hierbei in einzelnen Fällen auch über das Berliner Zentrum operierte. Bereits Anfang des Jahres 1935 hatte Gilbert MacMaster in einem Schreiben an Clarence Pickett auf die Probleme verwiesen, die sich aus der engen Zusammenarbeit zwischen Hughes einerseits und Catchpool und damit auch dem Berliner Zentrum andererseits vor allem für die dem Zentrum verbundenen deutschen Quäker ergaben: „What foreigners could probably do without danger to themselves, might mean imprisonment for German Friends and the dissolution of the German Society if a connection was established. “46 Als dann im August 1935, offenbar nach einem anonymen Hinweis, das Adressbuch der britischen Quäkerin Elizabeth Howard Fox an der deutsch-belgischen Grenze beschlagnahmt wurde, geriet das Zentrum erneut ins Visier der Gestapo. In dem Verzeichnis hatte Fox

AFSCA, General Files, Foreign Service, Country: Germany 1935 (Gilbert MacMaster Correspondence). Da er keine Antwort erhielt, richtete sich Catchpool gemeinsam mit Paul Sturge mittels eines offenen Briefes an Hitler. Erneut wurden die KZ, die Unterdrückung der politischen Opposition und die Verfolgung und Entrechtung von Minoritäten beklagt. Dies, so der Brief weiter, mochten innere Angelegenheiten sein, ,although our moral sense denies their validity in any and every case“. Was man erreichen wolle, ,is to press upon the German people the urgent necessity of looking at these measures in the light of their repercussion on the world“. Die dauerhafte Beibehaltung der Maßnahmen ,would be to dishonour a great nation. (...) to retain them longer must indefinitely postpone the hour of her entrance into that closer friendship for which we plead“, offener Brief „to the German chancellor and people“ 5/7/1935, in: LSRF, German Emergency Committee 1935 (ohne Signatur). Auch in der Folge bemühte sich Catchpool mit wechselndem Erfolg um persönliche Gespräche mit Rudolf Hess, Heinrich Himmler oder Ernst Hanfstängel, um sich für einzelne Persönlichkeiten wie von Ossietzky zu verwenden oder den Standpunkt der Quäker generell zu verdeutlichen.

45 In einem Schreiben des FSC an den Reichsführer SS Heinrich Himmler etwa wurde die Erlaubnis zum Besuch des Konzentrationslagers Papenburg erfragt: „Sie haben mehrmals in der Vergangenheit Quäkern die Erlaubnis erteilt KZ zu besuchen und die Art, wie die Insassen dort leben und arbeiten, kennen zu lernen (...). Wir sind überzeugt, dass die Besuche in Lichtenburg und Dachau viel zum Verständnis beigetragen haben, denn wir konnten dadurch vielen in England auf ihre besorgten Anfragen berichten, dass es ihren Freunden dort gut geht.“ FSC an Heinrich Himmler 22/5/1935, in: AFSCA, General Files, Foreign Service, Country: Germany 1935. 46 Gilbert MacMaster an Clarence Pickett 4/1/1935, in: AFSCA, General Files, Foreign Service, Country: Germany 1935 (Reports). 
Kontaktadressen von Gegnern des Regimes notiert sowie von Personen, die, vom Berliner Zentrum vermittelt, diesen Zuflucht boten. ${ }^{47}$

Hans Albrecht, der aufgrund eben dieser Aktivitäten ebenso wie Catchpool und einige weitere Mitarbeiter des Zentrums einige Tage inhaftiert war, appellierte deshalb einmal mehr mit amerikanischer Unterstützung an Catchpool, die Arbeit des Berliner Zentrums strikt vom „political relief“ zu trennen, wie ihn etwa das Londoner Emergency Committee leistete. Dies war auch ein guter Teil der Erklärung dafür, warum Albrecht schockiert reagierte, als MacMaster 1935 ankündigte, seine Tätigkeit im Zentrum zu beenden und dauerhaft in die Schweiz zurückzukehren. Ein etwaiger Rückzug der amerikanischen Quäker aus Berlin stelle „eine grosse Gefahr sowohl für die Aufrechterhaltung des Grundgedankens des Internationalen Sekretariats, als auch für die ganze Arbeit der Religiösen Gesellschaft der Freunde in Deutschland und Mitteleuropa“ dar. Man bitte daher „das American Friends Service Committee um die Aufrechterhaltung seiner Beteiligung und um möglichst baldige Wiederbesetzung der Stelle des amerikanischen Sekretärs“. ${ }^{48}$

Das AFSC dachte indes nicht daran, den Posten vakant zu lassen. Im Gegenteil: Auch das AFSC störte sich an der Art, wie Catchpool das Zentrum in Berlin führte. Schon im Januar 1935 hatte MacMaster Philadelphia darüber in Kenntnis gesetzt, dass von einer „internationalen“ Zusammenarbeit im Berliner Zentrum keine Rede sein könne. Vielmehr dominierten die Briten in nahezu allen Belangen. MacMaster sprach von einem Zustand der „non-cooperation“, für den er vor allem Catchpools Eigensinn verantwortlich machte. Das Fass zum Überlaufen brachte, dass dieser 1935 mit Unterstützung des FSC in London seine Amtszeit als Leiter des Berliner Zentrums eigenmächtig um ein weiteres Jahr verlängerte. Dies bedeutete einen Verstoß gegen die Übereinkunft turnusgemäßer Wechsel an der Spitze des Berliner Zentrums, dem nun eigentlich wieder ein Amerikaner hatte vorstehen sollen. Clarence Pickett war zur Verschiebung von Catchpools Ablösung nur bereit, wenn dieser ausdrücklich zusicherte, seinen Posten im April 1936 tatsächlich zu räumen. ${ }^{49}$ An MacMaster schrieb Pickett dazu: „Saintly and fine as Corder is, we know that his strong individualistic tendency practically means that

47 Bericht Hughes GEC und Holland an Germany 30/8/1935.

48 Protokoll einer Sitzung der Deutschen Jahresversammlung aus dem Jahr 1935, zitiert von Albrecht selbst drei Jahre später, als es erneut um die Wiederbesetzung der amerikanischen Stelle ging. Hans Albrecht an Paul Sturge (FSC) 8/6/1938, in AFSCA, General Files, Foreign Service, Country: Germany 1938 (Friends and Nazi Germany).

49 Clarence Pickett an Gilbert MacMaster 19/2/1935, in: AFSCA, General Files, Foreign Service, Country: Germany 1935 (Correspondence Gilbert MacMaster). 
the two people who are there go along parallel lines and that actually there is not much international cooperation. “50

Weitere Unstimmigkeiten kamen hinzu, wie aus der Korrespondenz zwischen Corder Catchpool und Clarence Pickett im Sommer 1935 hervorgeht, die sich um die Kampagne für die Freilassung Carl von Ossietzkys drehte. Catchpool beschwerte sich darüber, dass das AFSC diese in den USA durch die Presse publik mache, was dazu führe, dass Catchpool Anrufe amerikanischer Zeitungen abwehren müsse, die ihm Aussagen zur Verfolgung und den Konzentrationslagern entlocken wollten. Dies torpediere aus Catchpools Sicht den Erfolg seiner „stillen Diplomatie“. ${ }^{51}$ Pickett antwortete ausweichend, ließ jedoch durchblicken, dass er Catchpools Strategie nicht guthieß: „While it is true that the language which is used by a good many of the newspaper people would not be the language of Friends, it is perhaps the language which the persons to whom the letters are addressed understand better than much of the language which Friends would use." Damit signalisierte Pickett auch, dass aus Sicht des AFSC die Zeit vorbei sei, in der man auf eine Kooperation mit dem NS-Regime zu große Hoffnungen setzte. ${ }^{52}$

Das AFSC war deshalb mehr denn je entschlossen, das Heft im Berliner Zentrum in die eigene Hand $\mathrm{zu}$ nehmen. Vor diesem Hintergrund $\mathrm{zu}$ sehen war Ende 1935 die Berufung von Albert Martin, Professor für deutsche Literatur an der Brown University, zum designierten Nachfolger Catchpools. Martin - von Pickett als „young and vigorous“ angekündigt, und ausgestattet mit „all of the characteristics of the finest quality of Quakerly spirit of life“53 - war entschieden der Ansicht, dass sein Vorgänger die Quäker gefährlich nahe an das Regime herangeführt habe. Die Ernennung Martins, der sich noch in den USA für einen härteren Kurs gegenüber dem Regime ausgesprochen hatte, war damit ein deutliches Signal: In einem Brief an Clarence Pickett erklärte Martin kategorisch: „(...) there is

50 Clarence Pickett an Gilbert MacMaster 27/5/1935, in: AFSCA, General Files, Foreign Service, Country: Germany 1935 (Correspondence Gilbert MacMaster).

51 Speziell beschwerte sich Catchpool über einen Anruf von dem Korrespondenten der New York Times, der mit ihm am Telefon über politische Gefangene habe sprechen wollen und ihn dadurch in Verlegenheit gebracht habe, weil ja alles sehr genau von Gestapo beobachtet werde. Das sei alles schädlich und kontraproduktiv, und wenn überhaupt, solle alles auf die „non-antagonistic“ Weise der britischen Quäker geschehen. Corder Catchpool an Clarence Pickett 9/8/1935, in: AFSCA, General Files, Foreign Service, Country: Germany 1935 (letters from Germany).

52 Clarence Pickett an Corder Catchpool 19/9/1935, in: AFSCA, General Files, Foreign Service, Country: Germany 1935 (letters to Germany).

53 Clarence Pickett an Corder Catchpool 31/10/1935, in: AFSCA, General Files, Foreign Service, Country: Germany 1935 (letters to Germany). 
no compromise possible between the NS philosophy and our own“. ${ }^{54}$ Seine Ernennung stand damit für einen weitgehenden Kurswechsel: Je länger das NSRegime andauerte und je mehr Opfer es hervorbrachte, die sich mit der Bitte um Hilfe an die Quäker wandten, desto weniger schien eine ausgewogene Position gegenüber dem Regime gerechtfertigt. Die grundlegende Spannung blieb jedoch bestehen: Die Frage, ob es der angemessenere Quäker-Ansatz sei, alle Energie auf die Linderung der Not der Verfolgten zu legen, oder ob es trotz schrecklicher Verbrechen besser sei, gleich unter welchen Bedingungen, im Dialog mit den Tätern zu bleiben, blieb bis zum Schluss ungeklärt.

Unter Martins Führung gewann das Zentrum seinen Charakter als International Center zurück (wenngleich der Begriff „International“ selbst vorsorglich aus dem Namen gestrichen wurde), das heißt als Ort der Zusammenarbeit zwischen deutschen und ausländischen Quäkern. Die individuellen Hilfsaktionen über das Zentrum selbst wurden heruntergefahren - so wie Hans Albrecht es gefordert hatte. Martin und Douglas Steere reisten stattdessen durch Deutschland, um den persönlichen Kontakt mit den kleineren Zentren zu halten. Aus der Korrespondenz mit der Zentrale in Philadelphia - aus Gründen der Geheimhaltung verschickten Martin und andere Mitarbeiter des Zentrums Briefe mit Lageberichten in der Regel aus Kopenhagen oder von anderen Orten außerhalb Deutschlands wird seine Abscheu vor dem Nationalsozialismus ebenso deutlich wie die geringen Aussichten, die er für einen politischen Wechsel sah. ${ }^{55}$ Auch wenn die bedrängte Lage, in der sich nahezu alle verbliebenen Quäker und „friends of the Friends“ in Deutschland befanden, durchaus eine Rolle spielte, ist an Martins Briefen auffällig, das er religiösen Themen vergleichsweise wenig Gewicht gab. ${ }^{56}$

54 Albert Martin an Clarence Pickett 7/11/1936, zitiert in: Schmitt, Quakers and Nazis: Inner Light in Outer Darkness, 113.

55 So etwa in einem Brief Albert Martins, in dem er eine düstere Einschätzung zu den $\mathrm{Zu}$ kunftsaussichten des Regimes äußert, in dem „fanatics“ und die „most cruel, mercenary, unscrupulous elements of the country“ ihren Zugriff auf das Land immer weiter ausdehnten. Martins geringe Hoffnungen ruhten auf einer kleinen Schicht von Intellektuellen, die dem „tissue of falsehoods and falsifications“, mit dem das Regime das Land überzog, wenig entgegenzusetzen hatte. Albert Martin an Clarence Pickett 10/6/1937, in: AFSCA, General Files, Foreign Service, Country: Germany 1937 (Correspondence Berlin Centre).

56 Dies wurde deutlich vor allem im Kontrast zu den Briefen von Martins Frau Anne und von Douglas Steere, die ein sehr lebhaftes Bild sowohl des Gemeindelebens und der Bedrängnis unter dem Nationalsozialismus als auch zu spirituellen Aspekten boten. So etwa typisch in einem Schreiben Steeres, in dem er ein breites Bild über die Aktivitäten von Quäkern und Sympathisanten (etwa Pastor Wilhelm Mensching aus dem niedersächsischen Petzen, ein „friend of the Friends“ seit den 1920er Jahren und als Anhänger der Bekennenden Kirche wichtiger Verbindungsmann der Quäker in Deutschland) in ganz Deutschland bot. Douglas Steere an Clarence 
Vielmehr interpretierte er seine Funktion, wohl durchaus im Einklang mit den Vorstellungen des AFSC in Philadelphia, in erster Linie als Verwalter, dessen erste Aufgabe es war, den Fortbestand der Zusammenarbeit in Deutschland zu sichern.

Das Berliner Zentrum wurde unter Martins Führung ein Rad im größeren Getriebe der Arbeit für die Verfolgten des NS-Regimes, deren Schwerpunkt sich zunehmend nach außen, jenseits der deutschen Grenzen verlagerte. Während Catchpool nach Verlassen seinen Postens 1936 bis zum Ausbruch des Krieges immer wieder nach Deutschland zurückkehrte, um seine Kontakte in beide Richtungen, zu den Gegnern des Regimes ebenso wie zu Berber und anderen, aufrechtzuerhalten, wandte sich das AFSC nun auf allen Ebenen zunehmend denjenigen zu, die vor dem Terror und der Verfolgung bereits ins Ausland entflohen waren oder nach Wegen suchten, dies zu tun.

\section{Eine Infrastruktur der Hilfe. Das AFSC und die Flüchtlinge aus NS-Deutschland}

Damit gewann automatisch jene Hilfe an Bedeutung, die das AFSC seit 1933 leistete, um die Flüchtlingskrise zu bewältigen, die dem nationalsozialistischen Machtantritt gefolgt war. Hier unterstützte Philadelphia erstens die britischen Glaubensgenossen an den Brennpunkten der Flucht wie Paris oder Wien. Zweitens beteiligte sich das AFSC auch an internationalen Bemühungen, das Problem der Flüchtlinge aus Deutschland auf Regierungsebene und über den Völkerbund in Genf zu regeln. Schließlich erhielt drittens auch die Arbeit in den USA für die Aufnahme deutscher Flüchtlinge ein größeres Gewicht.

Ein Fokus der britischen Quäker seit 1933 hatte zunächst auf der Errichtung einer Infrastruktur der Hilfe für die deutschen Flüchtlinge gelegen, darunter vor allem für jene, die es nach Großbritannien geschafft hatten. Das Friends House in London beherbergte ab April 1934 ein eigenes Fallkomitee für Flüchtlinge aus Deutschland. Wie in Deutschland selbst bestand eine Arbeitsteilung mit jüdischen Organisationen, die im benachbarten Woburn House residierten: Während diese sich um jüdische Flüchtlinge kümmerten, bemühten sich die Quäker in erster Linie um nichtjüdische, vorwiegend um politische Flüchtlinge sowie die sogenannten nichtarischen Christen. Kooperationen bestanden mit einer Vielzahl weiterer, auf bestimmte Gruppen von Flüchtlingen konzentrierter Organisationen, die ihre Hilfe teilweise über die Quäker kanalisierten, darunter Save the Children,

Pickett (aus Bornholm) 15/8/1937 in: AFSCA, General Files, Foreign Service, Country: Germany (Correspondence Berlin Centre). 
der International Student Service oder der Academic Assistance Council. Die Quäker halfen mit Geldleistungen, aber auch bei der Suche nach Wohnraum oder Schulen für die Kinder. ${ }^{57}$

Aufgrund der restriktiven Einwanderungspolitik Großbritanniens bestand eine der Hauptaufgaben des Komitees darin, die Geflüchteten durch berufliche Schulungen auf ihre Auswanderung in andere Länder, vorwiegend in Nord- und Südamerika, vorzubereiten. Aus demselben Grund richtete sich ein Hauptaugenmerk der Arbeit des Emergency Committee von Beginn an auf die Arbeit im Ausland.

Das mit Abstand wichtigste Zentrum der Hilfe lag in Frankreich, wo die Mehrheit der Flüchtlinge aus Deutschland strandete. Hier lief der Großteil der Hilfe zunächst über L'Entre Aide Européene, eine Organisation, die 1931 von französischen Quäkern gegründet worden war, um das französisch-deutsche Verständnis zu fördern. 1934 wurde ihre Arbeit in einen Service International d'Aide aux Refugies de la Societé des Amis überführt, der am Sitz der Pariser „Quäkerbotschaft“ im Quartier Latin untergebracht war. Der Service International, der als gemeinschaftliches Werk französischer Quäker, des AFSC und des FSC agierte, diente zu dieser Zeit jährlich Tausenden von deutschen Flüchtlingen als erste Anlaufstelle. ${ }^{58}$ Ähnlich wie das Londoner Emergency Committee arbeitete der Service International auf Fallbasis, und kümmerte sich um die unmittelbaren Bedürfnisse der Flüchtlinge. Er organisierte Kleiderspenden und provisorische Unterkünfte sowie eine medizinische Grundversorgung, unterhielt eine Kantine und einen Kindergarten. In Frankreich, wo die Flüchtlinge auf dem Arbeitsmarkt im Vergleich zu England weniger hohe Hürden vorfanden, spielten deshalb neben beruflichen Schulungen und landwirtschaftlicher Ausbildung ${ }^{59}$ auch Sprachkurse eine bedeutende Rolle, da die wenigsten Menschen bei ihrer Ankunft über genügend Französischkenntnisse verfügten.

Auch in Wien und Prag dienten die jeweiligen Quäkerzentren als Zufluchtsort für jeweils mehrere Hundert Menschen, die aus Deutschland geflohen waren. Insbesondere die Wiener „Quäkerbotschaft“, die wie das Zentrum in Berlin als Internationales Zentrum unter angloamerikanischer Führung stand, sollte in den folgenden Jahren eine bedeutende Stellung im Netzwerk der Quäker-Flücht-

57 Darton, An Account of the Friends Committee for Refugees and Aliens, First Known as the Germany Emergency Committee of the Society of Friends, 1933-1950. 34-44.

58 Ein Überblick über diese Arbeit in AFSC Annual Report 1936, 13.

59 Zur landwirtschaftlichen Ausbildung etablierte der Service International mit Unterstützung des GEC eine Modellfarm in Le Mosset in den französischen Ost-Pyrenäen, in: GEC-Bulletin May (1934). Darton, An Account of the Friends Committee for Refugees and Aliens, First Known as the Germany Emergency Committee of the Society of Friends, 1933-1950, 16-28. 
lingshilfe erlangen. Das Londoner Komitee kümmerte sich daneben auch um eine Reihe weiterer Initiativen wie das erwähnte Rest Home für vormalige KZ-Insassen und deren Angehörige im Taunus. Ein besonderes Gewicht lag auch auf zwei Schulprojekten in den Niederlanden - in Eerde/Ommen und Haarlem -, die in erster Linie Kinder aus deutschen Familien aufnahmen, die geflohen waren oder ihre Kinder dem nationalsozialistischen Schulsystem entziehen wollten. ${ }^{60}$

Nach und nach wurden die verbliebenen vier Quäkerzentren in Europa, in Berlin, Wien, Paris und Genf zu Knotenpunkten der Flüchtlingshilfe. Die AFSCBulletins dieser Jahre spiegeln diesen Übergang gut wider. Während zu Anfang noch Berichte dominierten, die die Rolle der Zentren als Orte spiritueller Erneuerung betonten, nahmen die Berichte zur Flüchtlingsarbeit ab 1934 immer größeren Raum ein. ${ }^{61}$ Parallel richtete auch das AFSC seine Aufmerksamkeit zunehmend auf diese Frage, sowohl im Ausland als auch in den USA selbst. Wohlgemerkt: An all den eben genannten Projekten war das AFSC von Anfang an beteiligt. Immer wieder entsandte das Komitee Delegationen nach Deutschland und in die europäischen Länder, in denen sich die deutschen Flüchtlinge konzentrierten, um den Bedarf und die Möglichkeiten für Hilfsmaßnahmen zu ermitteln. Meist leistete das Komitee zunächst finanzielle Unterstützung, während die Organisation in den Händen des German Emergency Committee in London lag. Eine der Arbeit des britischen GEC vergleichbare Flüchtlingspolitik im eigenen Land betrieb das AFSC bis 1935 nur in Einzelfällen. ${ }^{62}$ Eine grundsätzliche Ände-

60 Die Schule in Eerde/Ommen wurde den Quäkern 1934 von seinem Besitzer, dem Grafen Philipp von Pallandt Eerde überlassen, der in der Zeit nach dem Ersten Weltkrieg mit den Quäkern in Berührung gekommen war. Unter den Lehrerinnen und Lehrern der Schule befanden sich viele deutsche Exilanten. Prinzipal der Schule war die Hamburger Reformpädagogin Katharina Petersen. An der Etablierung der Schule war der Vorsitzende der Quäkergemeinde in Deutschland, Hans Albrecht, beteiligt. Auch bei der Home School Haarlem handelte es sich um ein Projekt der den Quäkern lange verbundenen Reformpädagogen Karl und Lilli Pollatz, in dem viele deutschjüdische Flüchtlingskinder unterkamen. Beide Schulen mussten nach der deutschen Besatzung ihren Betrieb einstellen oder den geänderten Umständen anpassen und die jüdischen Kinder größtenteils in Verstecken unterbringen (neun der Kinder aus Eerde wurden im Jahr 1943 deportiert und schließlich im KZ ermordet, für Haarlem sind die Zahlen nicht bekannt). Karl Pollatz wurde unter deutscher Besatzung verhaftet. Schmitt, Quakers and Nazis: Inner Light in Outer Darkness, 29-30; Bob Moore, Refugees from Nazi Germany in the Netherlands, 1933-1940, Studies in Social History 9 (Dordrecht: Martinus Nijhoff Publishers, 1986).

61 Annual Report 1935, in: AFSC-Bulletin 97 (1936); Annual Report 1936, in: AFSC-Bulletin 104 (1937).

62 So hatte das AFSC im August 1933 seine bestehenden Kontakte zu nutzen versucht, um eine Einreise von Thomas Mann in die USA zu ermöglichen. Ray Newton (Secretary Peace Section) an Cordell Hull 17/8/1933; Cordell Hull an Ray Newton 19/8/1933, in: AFSCA, General Files, Foreign Service, Country: Germany 1933. 
rung trat abermals erst nach Verkündung der Nürnberger Rassengesetze ein, die die jüdische Bevölkerung weiter aus dem öffentlichen Leben ausgrenzten und so neue Fluchtbewegungen aus Deutschland auslösten. Parallel zur Ernennung Albert Martins zum neuen Leiter des Berliner Zentrums reagierte die AFSC-Zentrale in Philadelphia auf die Entwicklungen unter anderem mit der Einrichtung einer eigenen Refugee Section, die von Robert Yarnall geleitet wurde. Zusätzlich übernahm Hertha Kraus, die sich seit 1934 in den USA aufhielt und seit 1935 als Professorin für Sozialwirtschaft am Quäker-(Frauen-)College Bryn Mawr (bei Philadelphia) lehrte, zunächst ehrenamtlich und ab 1937 als regulärer Teil des AFSC die Funktion einer Beauftragten für die Flüchtlinge aus Deutschland. ${ }^{63}$ Das AFSC legte neues Gewicht darauf, die Voraussetzungen für eine erleichterte Immigration deutscher Flüchtlinge in die USA zu schaffen und kümmerte sich etwa um die notwendigen eidesstattlichen Erklärungen für individuelle Fälle. ${ }^{64}$ Kraus' Stelle betrieb dabei case work für eine ausgewählte Gruppe von Flüchtlingen und bemühte sich über die Grenzen der USA hinaus auch um alternative Zufluchtsorte. ${ }^{65}$ Bereits seit 1933 befand sich das AFSC hier in einem Dialog mit leitenden Vertretern der jüdischen Gemeinschaft in den USA über eventuelle jüdische Kolonien vor allem in lateinamerikanischen Ländern. ${ }^{66}$ Konkreter wurden solche Planungen später mit Bezug auf Kolumbien ${ }^{67}$ und Kuba, ohne letztlich zu einem greifbaren Ergebnis zu führen. ${ }^{68}$

Bei all diesen Aktivitäten für die Opfer des nationalsozialistischen Terrors und insbesondere für diejenigen, die entweder Deutschland verlassen wollten oder bereits aus dem Land geflohen waren, waren die Quäker in den USA mit denselben grundlegenden Schwierigkeiten konfrontiert wie ihre europäischen Kollegen: Der Einwanderung standen hohe Hürden entgegen, denn die entspre-

63 AFSC Annual Report 1938, 13-14.

64 Report on the mission of Alfred Scattergood to Germany 1937, in: AFSCA General Files, Foreign Service Germany 1937 (Germany General).

65 Hertha Kraus, International Relief in Action, 1914-1943: Selected Records, with Notes (Scottdale, PA: The Herald Press, 1944).

66 Ein Bericht über ein solches Treffen im Mai 1933 findet sich erstmals in der Korrespondenz zwischen Clarence Pickett und Bertram Pickard. Die Idee jüdischer Kolonien wurde in der Folge immer wieder aufgegriffen. Clarence Pickett an Bertram Pickard 17/5/1933, in: AFSCA, General Files, Foreign Service, Country: Germany 1933 (Bertram Pickard, Geneva).

67 Siehe etwa Preliminary Notes (strictly confidential) on the Possibilities of settling German Refugees on the Land in Colombia (July 1935), in: AFSCA, Foreign Service, Country: Germany 1935 (German Refugees General).

68 Die AFSC-Archive enthalten etwa einen ausführlichen „Kolonisierungsplan“ für Lateinamerika, „Colonization plan“ 8/8/1935, in: AFSCA, Foreign Service, Country: Germany 1935 (German Refugees General). 
chende Politik der USA war wie in Großbritannien im Gefolge der Weltwirtschaftskrise noch restriktiver gestaltet worden, als sie ohnedies bereits seit dem Ende des Ersten Weltkriegs gewesen war. Insbesondere der Einwanderung von jüdischen Flüchtlingen standen eine Reihe von ausgesprochenen oder unausgesprochenen Vorbehalten entgegen. Für eine Erhöhung der Einwanderungsquote aus Deutschland (und Österreich), die den Flüchtlingen zugutegekommen wäre, fand sich ebenso wenig eine Mehrheit wie für eine auf bestimmte Zielgruppen (etwa Juden, politisch Verfolgte oder Kinder) ausgerichtete Ausnahmeregelung.

Das AFSC betrieb im Angesicht dieser Hindernisse ab 1935 auch zunehmend Lobbyarbeit bei der eigenen Regierung, um die erheblichen gesetzlichen Hindernisse, die der Einwanderung entgegenstanden, abzubauen. Es übernahm eine Führungsrolle in der amerikanischen Kampagne für deutsche Flüchtlinge und begann zu diesem Zweck eine enge Zusammenarbeit mit dem liberal-protestantischen Federal Council of Churches sowie jüdischen Wohlfahrtsorganisationen wie dem JDC. Damit beteiligte sich das AFSC auch am Aufbau einer Infrastruktur zur Aufnahme und Integration der Einwanderer. ${ }^{69}$ Der Fokus lag dabei wie in Großbritannien auf der Gruppe der Non-Aryan Christians. ${ }^{70}$ Wiederholt versuchte Clarence Pickett auch seine Regierungskontakte für eine Verbesserung der Lage einzusetzen, im Angesicht der Widerstände im Kongress allerdings ein schwieriges Unterfangen. Bei den rund 150 Gelegenheiten, bei denen Pickett während der Roosevelt-Jahre im Weißen Haus oder der privaten Residenz des Präsidenten in Hyde Park weilte - zumeist waren dies Treffen mit Eleanor Roosevelt, die sich um innenpolitische Fragen wie die homesteads drehten -, kam Deutschland immer wieder zur Sprache. ${ }^{71}$

Parallel hierzu gewannen nun auch die internationalen Aktivitäten zur deutschen Flüchtlingskrise ein immer größeres Gewicht. Bereits früh war das AFSC neben dem britischen FSC in die Bemühungen des Völkerbundes eingebunden. Im Oktober 1933 hatte dieser ein Hochkommissariat für die Flüchtlinge aus Deutschland unter dem Vorsitz des US-amerikanischen Diplomaten James G. McDonald eingerichtet. Diese Behörde arbeitete allerdings unter widrigen Bedingungen. Zunächst blieb Nazi-Deutschland, trotz seines bereits 1933 angekündigten Austritts aus dem Völkerbund, formal noch weitere zwei Jahre Mitglied mit

69 Michael Luick-Thrams, Out of Hitler's Reach: The Scattergood Hostel for European Refugees 1939-1943 (Mason City, IA: Michael Luick-Thrams, 1996). Zur protestantischen Antwort siehe: William E. Nawyn, American Protestantism's Response to Germany's Jews and Refugees, 1933-1941 (Ann Arbor: UMI Research Press, 1981).

70 Foreign Service Work, in: AFSC-Bulletin 104 (1937).

71 Lawrence McK. Miller, Witness for Humanity: A Biography of Clarence E. Pickett (Wallingford, PA: Pendle Hill Publications, 1999), 144. 
allen Rechten. So scheiterte der ursprüngliche Plan, das bestehende Flüchtlingskommissariat des Völkerbundes (das Nansen-Amt) mit der Frage der deutschen Flüchtlinge zu betrauen, am Widerstand Deutschlands. McDonalds Stelle agierte damit in einer Art Graubereich: Angesiedelt unter dem Dach des Völkerbundes, ohne jedoch ein offizieller Teil der Organisation zu sein, fehlten ihm sowohl die politische Unterstützung als auch die materiellen Mittel. ${ }^{72}$ Damit war das Hochkommissariat auf die Hilfe privater Organisationen angewiesen. Hier kamen die Quäker ins Spiel. Anfang 1934 wurden das AFSC und der britische FSC gemeinsam mit einer Reihe anderer, zumeist jüdischer Organisationen Teil des von McDonald einberufenen Beirats, der die privaten Hilfsmaßnahmen zugunsten der deutschen Flüchtlinge koordinieren sollte. ${ }^{73}$ Eine Broschüre, welche die britischen Quäker 1935 herausgaben, brachte es auf den Punkt: „A high commission for Refugees from Germany has been set up by the League of Nations, but the Funds to help the refugees must come from generous givers. “74

Durch die Mitarbeit im McDonald-Komitee sah sich das AFSC vor eine Reihe von Problemen gestellt. Während ein Teil der britischen Quäker den Völkerbund als ein zusätzliches Medium begriff, um Druck auf ihre eigene Regierung zugunsten der deutschen Flüchtlinge auszuüben, war dies für den AFSC aufgrund der Nicht-Mitgliedschaft der USA schwierig. Darüber hinaus belebte die quasioffizielle Verbindung der Quäker mit dem Völkerbund aufs Neue die Diskussionen um die „richtige“ Haltung der Quäker zu den durch den Völkerbund vertretenen Prinzipien kollektiver Sicherheit.

Hier war das AFSC, wie andere Mitglieder der Friedensbewegung in den USA, gefangen zwischen einem grundsätzlichen Internationalismus und einer quasinatürlichen Nähe zu isolationistischen Strömungen, die sich aus dem Ziel ergaben, ein Engagement der USA in einem neuen kriegerischen Konflikt in Europa zu verhindern. Das AFSC war Teil der breiten Front, die hinter der Verabschiedung des Neutrality act von 1935 stand, durch den der Kongress die Einmischung in auswärtige Konflikte weiter erschwerte (wie im folgenden Kapitel zum Spanischen Bürgerkrieg noch weiter auszuführen sein wird). Pickett und andere im AFSC gehörten zu den Hauptinitiatoren der Emergency Peace Campaign, die ab 1935 als Sammlungsbewegung der amerikanischen Friedensbewegung in Reaktion auf die

72 Fritz Kieffer, Judenverfolgung in Deutschland - Eine Innere Angelegenheit? Internationale Reaktionen Auf Die Flüchtlingsproblematik 1933-1939 (Historische Mitteilungen/Beiheft 44) (Stuttgart: Franz Steiner, 2002).

73 James McDonald an Clarence Pickett 24/2/1934, in: AFSCA, General Files, Foreign Service, Country: Germany 1934 (High Commissioner for Refugees from Germany).

74 Broschüre German Refugee Crisis, in: LSRF, German Emergency Committee 1935 (ohne Signatur). 
sich zuspitzende Lage in Europa entstand. Der Vorsitzende der peace section Ray Newton diente als Executive Director der Kampagne und Pickett nutzte auch in diesem Fall seine Kontakte, um Eleanor Roosevelt für Redebeiträge zu gewinnen. ${ }^{75}$

Eine Frage, die innerhalb der Bewegung kontrovers diskutiert wurde, war, inwieweit nichtmilitärische internationale Maßnahmen gegen Deutschland, das heißt Boykotte oder Sanktionen, über den Völkerbund gerechtfertigt waren. In dieser Frage war die Friedensbewegung gespalten, was auch auf das AFSC zutraf, dessen Mehrheit jedoch zu einer Ablehnung neigte. Clarence Pickett war keine Ausnahme: Im Dezember 1934 schrieb er in diesem Sinn an Gilbert MacMaster und berichtete über den Beginn einer großen Hilfskampagne in den USA zugunsten der Opfer der Verfolgung in Deutschland, für die unter anderen dem AFSC nahestehende Persönlichkeiten wie Jane Addams und der Erziehungswissenschaftler John Dewey warben. Das AFSC hatte, wie Pickett berichtete, eine Zusammenarbeit mit der Kampagne abgelehnt, weil in ihren Aufrufen scharfe Anklagen gegen das Nazi-Regime erhoben wurden und die Spendenkampagne mit Boykottaufrufen gegen Deutschland kombiniert war. Man habe „definitely refused to cooperate with efforts which imply that the use of emotion of hate or bitterness is ever justifiable“. Pickett war überzeugt, „that the use (...) of personal or group boycott is not sound and should never be used“. ${ }^{76}$ Auch rund zwei Jahre später wich Pickett von dieser Linie nicht ab. Den Aufruf der Non-Sectarian AntiNazi-League, die sich im Vorfeld der Berliner Olympischen Spiele 1936 für einen Reiseboykott gegenüber dem Reich aussprach, beantwortete der Generalsekretär auf die gleich kategorische Weise. Gegenüber Deutschland, einem Land „so definitely suffering from an inferiority complex - a complex we as one of the allies have largely induced through our treatment of Germany after the war" -, sei der Boykott das falsche Mittel: Es sei „increased circulation that is needed rather than decreased circulation“, um freundliche Beziehungen und Vertrauen zwischen den Völkern herzustellen. ${ }^{77}$

Zur gleichen Zeit vertrat das AFSC nach außen immer offener eine pro-internationalistische Position. Dies ließ sich insbesondere an der deutlich positiven Haltung zum Völkerbund ablesen, die einen deutlichen Kontrast nicht nur zur isolationistischen Tendenz im eigenen Land, sondern auch $\mathrm{zu}$ den britischen

75 Miller, Witness for Humanity: A Biography of Clarence E. Pickett., 153-155. Cortright, Peace: A History of Movements and Ideas, 85-87.

76 Clarence Pickett an Gilbert MacMaster 13/12/1934, in: AFSCA, General Files, Foreign Service, Country: Germany 1934 (letters from Gilbert MacMaster).

77 Clarence Pickett an G. E. Harriman (Non-Sectarian Anti-Nazi League) 2/3/1936, in: AFSCA, Foreign Service, Country: Germany 1936. 
Quäkern darstellte. ${ }^{78}$ Einen guten Indikator stellten auch die intensivierten Beziehungen zum Genfer Internationalen Zentrum dar, dessen Arbeit in den Publikationen des AFSC viel Raum gegeben wurde. „In a time when nationalism is prevalent“, erklärte ein Artikel im AFSC-Bulletin 1934, „it seems doubly important to keep open these channels of contact and understanding between (...) European Centers and our American life." 79 Das Zentrum unter Bertram Pickards Leitung erschien dabei nicht nur als Quelle internationaler Nachrichten, sondern wurde selbst als quasi-diplomatischer Arm der Quäker in der Weltpolitik porträtiert. So hieß es etwa im Jahresbericht des AFSC 1935 vor dem Hintergrund des italienischen Überfalls auf Äthiopien, das Genfer Zentrum „has been occupied very fully during the later months of the year in efforts to make effective the peace machinery of the world in the Italo-Ethiopian conflict““ ${ }^{80}$ Ein reger Briefwechsel zwischen Philadelphia und Genf wurde durch eine intensive Reisetätigkeit Gilbert MacMasters ergänzt, der von seinem Baseler Wohnsitz aus häufig nach Genf fuhr und Bericht über die Sitzungen des Völkerbundes und internationale Fragen erstattete. ${ }^{81}$

1937 wurde die Genfer Stelle weiter aufgewertet, als das Quäkerzentrum ins vom Völkerbund aufgegebene Palais Wilson zog, ins Zentrum des internationalen Lebens. Das AFSC-Bulletin kommentierte den Umzug und lobte die Arbeit Pickards, der höchstes Gewicht verliehen wurde, beruhe diese Arbeit doch auf der festen Überzeugung von der ,continued vitality and fruitful activity of the League of Nations“, insbesondere mit Blick auf die „valuable social and economic work (that) continues to be done“. Angesicht der schwierigen internationalen Lage befinde sich der Völkerbund politisch gegenwärtig „under a cloud“. Umso bedeutender sei die Arbeit der Quäker in Genf, die sich an der Spitze derjenigen befänden, die helfen wollten eine „new and better League“ zu errichten. ${ }^{82}$

Der entschiedene und sich als im Quäkersinn konstruktiv definierende Internationalismus des AFSC zeigte sich zur selben Zeit auch an anderen Stellen. Vor allem an der breitgefächerten Friedensarbeit der peace section lässt sich dies nachverfolgen: In den „Friedenskarawanen“, den Institutes of International Relations für Studenten, den organisierten Reisen nach Genf und der Arbeit zur

78 Einen guten knappen Überblick über die Positionen mit Bezug zur Friedensbewegung auf beiden Seiten des Atlantiks bietet die Einleitung von Farah Mendlesohns Buch zum Spanischen Bürgerkrieg, Farah Mendlesohn, Quaker Relief Work in the Spanish Civil War (New York: Edwin Mellen Press, 2002), 7-23.

79 Annual Report 1934, in: AFSC-Bulletin 94 (1935).

80 Annual Report 1935, in: AFSC-Bulletin 97 (1936).

81 Waugh, „Quakers, Peace and the League of Nations: The Role of Bertram Pickard.“

82 Creative Experience in International Relations, in: AFSC-Bulletin 98 (1936). 
Friedenserziehung über die Schullehrpläne - überall nahm die Aufklärung über den Völkerbund eine zentrale Stellung ein. ${ }^{83}$ Die Arbeit zielte darauf ab, das Bewusstsein für das friedenspolitische Potenzial des Völkerbundes (und den Nutzen einer Mitgliedschaft der USA) zu schärfen. Schließlich bedeutete dieser internationalistische Schwenk noch in einer weiteren Hinsicht eine Neu- beziehungsweise Re-orientierung. Im gleichen Maße, in dem die Flüchtlingsarbeit in den Vordergrund der Arbeit in Philadelphia und den europäischen Zentren rückte, nahm der Internationalismus des AFSC zunehmend praktische Züge an und das AFSC war auf dem besten Weg, in gewisser Weise zu seinen Anfängen als internationaler Hilfsorganisation zurückzukehren. Dieser Prozess verlief allerdings schleichend und der Weg dorthin war keineswegs vorgezeichnet. 1935 betonte das AFSC-Bulletin noch entschieden, der Hauptzweck der Arbeit in den europäischen Zentren bestehe darin, „to interpret American life to European countries and European life to America“. Es handele sich dabei, so betonte der Artikel trotz aller momentaner Erfordernisse durch die Flüchtlingshilfe um einen primär „spiritual service“. Auch das Berliner Zentrum wurde in erster Linie als ein Ort porträtiert, an dem „troubled souls find consolation““ ${ }^{84}$ Selbst zwei Jahre später wurde die Bedeutung der europäischen Arbeit in Abgrenzung zur Nachkriegszeit noch als vorwiegend spirituell beschrieben: Ein europäischer Quäker wurde im affirmativen Sinn mit den Worten zitiert: ,you felt the concern to go out and feed starving children 15 years ago. May it be your concern now to go out and feed starving minds. "85 Selbst als die Hilfe für die Flüchtlinge bereits auf Hochtouren lief, entsandte das AFSC „Quaker ambassadors“ in verschiedene Teile der Welt. Rufus Jones bereiste im Jahr 1938 unter anderem Südafrika, China und Deutschland mit dem ausgewiesenen Ziel, sich den „spiritual needs“ der Menschen in diesen „troubled spots“ zu widmen. ${ }^{86}$

Die Betonung des nach wie vor spirituellen Charakters der Arbeit im Angesicht der wachsenden praktischen Anforderungen hatte dabei wohl unterschiedliche Gründe. Sie entsprach fraglos der ehrlichen Überzeugung vieler im AFSC, worin der eigentliche Beitrag der Quäker in der Weltpolitik liegen sollte. Die Betonung des spirituellen Kerns der Arbeit erfüllte im zeitlichen Kontext aber auch einen spezifischen Zweck, dann nämlich, wenn es galt das AFSC gegen den Vorwurf abzuschirmen, die Flüchtlingshilfe verletze die Neutralität der Quäker. Zudem bewegte sich das AFSC auch in der Frage der Einwanderung deutscher Flüchtlinge auf politisch vermintem Terrain.

83 Creative Experience in International Relations, in: AFSC-Bulletin 98 (1936).

84 Friends Service Abroad, in: AFSC-Bulletin 97 (1936).

85 Foreign Service, in: AFSC-Bulletin 111 (1938).

86 Annual Report 1938, in: AFSC-Bulletin 127 (1939). 


\section{Auf der Suche nach der right line of service. Das AFSC und die Fluchthilfe vor dem Zweiten Weltkrieg}

Das Jahr 1938 gab den letzten Anstoß für eine entschieden praktische Wende. „The present turmoil in international affairs has swept the needy and distressed to the doors of our four centres and thrown many new problems into the paths of our representatives“, hieß es dazu im Jahresbericht des AFSC. ${ }^{87}$ Der „Anschluss“ Österreichs im März und die folgende Welle des Terrors, die Annexion des tschechischen Sudetenlands im Gefolge der Münchner Konferenz im September sowie die Novemberpogrome in Deutschland selbst waren Wegmarken, die eine erneute Zuspitzung der Flüchtlingssituation nach sich zogen und weltweit den Sinn für die Dringlichkeit des Problems schärften.

Gleichzeitig zeigte die von den USA initiierte internationale Konferenz im französischen Evian im Sommer, dass die Bereitschaft der Regierungen gering war, für eine umfassende Lösung des deutschen Flüchtlingsproblems notwendige Konzessionen bei ihrer Einwanderungspolitik zu machen. Zwischen 1933 und dem Ausbruch des Krieges zögerten insbesondere Großbritannien und die USA, eine bedeutende Anzahl von Flüchtlingen aufzunehmen. Wenngleich Großbritannien nach 1938 seine Einreisebeschränkungen für deutsche Juden lockerte, entsprach die Aufnahmewilligkeit auch danach bei weitem nicht dem Bedarf. Zur selben Zeit schlossen sich in Palästina die Tore, da die britische Mandatsmacht auf arabischen Widerstand gegen die jüdische Einwanderung mit einer restriktiveren Politik antwortete. In Frankreich, wo die Einwanderungspolitik lange Zeit großzügiger gewesen war, bedeutete das Ende der 1930er Jahre mit dem parallelen Zustrom von Flüchtlingen aus Spanien, wo seit 1936 ein Bürgerkrieg tobte, ebenfalls eine Wende hin zu mehr Restriktionen. Mit anderen Worten: Türen schlossen sich weltweit in dem Moment, als der Bedarf am größten wurde. ${ }^{88}$

Die Evian-Konferenz änderte an diesem Umstand kaum etwas. Das Intergovernmental Committee aus 32 Staaten, das sich in der Folge gründete, brachte an einigen Stellen Erleichterungen, ohne für den im Lichte der weiteren Ereignisse in den Jahren 1938 und 1939 notwendigen Durchbruch zu sorgen. ${ }^{89}$

87 Annual Report 1938, in: AFSC-Bulletin 127 (1939).

88 Peter Gatrell, The Making of the Modern Refugee (Oxford: Oxford University Press, 2013), 73-76; John Mendelsohn, ed. Jewich Emigration from 1933 to the Evian Conference of 1938 (the Holocaust: Selected Documents in 18 Volumes/5) (New York/London: Garland, 1982).

89 Kieffer, Judenverfolgung in Deutschland - Eine Innere Angelegenheit? Internationale Reaktionen Auf Die Flüchtlingsproblematik 1933-1939 (Historische Mitteilungen/Beiheft 44); Tommie Sjöberg, The Powers and the Persecuted: The Refugee Problem and the Intergovernmental Com- 
Das AFSC verfolgte die Entwicklungen aufmerksam. Mit Myron Taylor, dem amerikanischen Delegierten auf der Evian-Konferenz, unterhielt Pickett regelmäßigen Kontakt. ${ }^{90}$ Auf die Entwicklungen reagierte das AFSC, indem es seinen Arbeitsschwerpunkt nun noch stärker auf jene richtete, die dem Terror des Regimes durch Flucht ins Ausland bereits entkommen waren, sowie auf diejenigen, für die dieser Schritt zu einer immer dringenderen Notwendigkeit wurde. Die Lage, die sich nach dem „Anschluss“ Österreichs an das Deutsche Reich im März 1938 entwickelte, gab dafür einen wichtigen Anstoß. Binnen weniger Tage sah sich das Wiener International Center mit den Folgen der unmittelbar einsetzenden Welle der Gewalt und der Verfolgung der jüdischen Bevölkerung und der politischen Gegner der Nationalsozialisten konfrontiert. ${ }^{91}$ Bis $\mathrm{zu}$ diesem Zeitpunkt hatte das von Briten und Amerikanern gemeinschaftlich geführte Zentrum im Ersten Wiener Bezirk (Singerstrasse) nur bedingt als Anlaufstelle für Flüchtlinge aus Deutschland gedient. Nun suchten in den ersten Tagen nach dem Anschluss bereits Hunderte von Verfolgten Schutz und Zuflucht vor dem Terror. Innerhalb kurzer Zeit wurde die Wiener Quäkerbotschaft zu einem Zentrum der Auswanderung und so zu einem Ort der Hoffnung und Zuflucht für jene, „who are compelled to live in suicide world“, wie der AFSC Jahresbericht für 1938 hervorhob. ${ }^{92}$

Für das AFSC übernahm Emma Cadbury, die bereits seit 1924 im Wiener Zentrum tätig war, die Koordination. Gemeinsam mit einer Handvoll Mitarbeiter (in der Regel acht bis zehn) behandelte das Zentrum von März 1938 bis zum Kriegsbeginn im September 1939 rund 11.000 Anträge auf Ausreise für ca. 15.000 Personen. Im Sommer 1938 half der Vorsitzende der Flüchtlingssektion des AFSC in Philadelphia Robert Yarnall selbst für zwei Monate im Zentrum aus. Die Hauptaufgabe bestand in der Suche nach aufnahmewilligen Ländern und Bürgen für die Ausreisewilligen sowie in der Erledigung der Formalitäten. Das Zentrum

mittee on Refugees (Icgr), 1938-1947 (Lund Studies in International History 26) (Lund: Lund University Press, 1991).

90 Taylor war als Vorstandsvorsitzender der United States Steel Cooperation Anfang der 1930er Jahre mit Pickett und dem AFSC im Rahmen der homestead-Bewegung in Berührung gekommen. Miller, Witness for Humanity: A Biography of Clarence E. Pickett., 147; 160 - 161; 171.

91 Unter dem Eindruck der Ereignisse veränderte sich der Charakter der Arbeit des britischen Quäkerhilfswerks in London. Das Emergency Committee wurde nun zusammen mit anderen großen jüdischen und christlichen Organisationen halbautonomer Teil eines umfassenden Flüchtlingsdienstes, der im Bloomsbury House eingerichtet wurde. In diesem Sinne wurde es in den letzten beiden Jahren vor dem Zweiten Weltkrieg weniger quäkerspezifisch, verfügte jedoch gleichzeitig über mehr Mittel für seine Arbeit. Darton, An Account of the Friends Committee for Refugees and Aliens, First Known as the Germany Emergency Committee of the Society of Friends, 1933-1950, 2-4; 58-61.

92 AFSC Annual Report 1938, 9. 
arbeitete eng mit den Konsulaten potenzieller Einwanderungsländer sowie mit jüdischen Organisationen zusammen. Es gelang dem Zentrum, etwa 4500 Menschen zur Ausreise zu verhelfen, wobei die meisten nach England gelangten, nur 300 in die USA. Mit den Behörden und der Gestapo bestand ein Abkommen, das es dem Zentrum erlaubte, weitgehend ungehindert $\mathrm{zu}$ arbeiten. Wie in Berlin konzentrierte sich die Arbeit des Zentrums insbesondere auf politisch Verfolgte und „nichtarische“ Christen, während sich die jüdische Kultusgemeinde den jüdischen Flüchtlingen widmete. Teilweise überlappten sich die Aktivitäten jedoch. So half das Wiener Zentrum etwa beim Unterhalt einer Schule für 150 Personen (der sogenannten Kagran-Gruppe), die in einem Vorort eine landwirtschaftliche Ausbildung erhielten, die sie auf die Ausreise (nach Palästina oder nach England) vorbereiten sollte. Die Quäker spielten darüber hinaus auch eine herausgehobene Rolle im Rahmen der sogenannten Kindertransporte. Das Zentrum organisierte die Ausreise von Kindern, deren Eltern sich in Gefängnissen und Konzentrationslagern befanden, oder die das Land aus anderen Gründen nicht verlassen konnten, und vermittelte ihre Aufnahme in Familien im Ausland. Vor Kriegsbeginn organisierte das Wiener Zentrum rund 1500 (von insgesamt etwa 10.000) solcher „Kindertransporte“ aus Österreich. Die Initiative lag wie schon nach dem Ersten Weltkrieg vor allem bei den britischen Quäkern, namentlich dem GEC in London. Wie auch in Berlin übernahm das AFSC mit fortlaufender Dauer jedoch einen größeren Teil der Arbeit, und angesichts der wachsenden Kriegsgefahr wurden die US-amerikanischen Quäker noch aktiver, auch um im Fall eines Kriegsausbruchs zwischen Deutschland und Großbritannien nahtlos von den Briten übernehmen zu können. ${ }^{93}$

Clarence Picketts Reise nach Europa, die ihn und seine Frau im September 1938 unter anderem nach Berlin, Prag und Wien führte, bestätigte einmal mehr die Dringlichkeit der Lage. Die Spuren der verschärften Verfolgung waren bereits auf der ersten Station, der Quäkerschule in Eerde/Ommen in Holland greifbar, da ihre Aufnahmekapazitäten bereits an ihre Grenzen gerieten. In Deutschland selbst waren Anspannung und Verzweiflung dann allgegenwärtig: angefangen beim amerikanischen Konsulat, das den Ansturm der Ausreisewilligen kaum bewältigen konnte, außerdem in den Gesprächen mit deutschen Quäkern, von denen

93 Das britische GEC gemeinsam mit weiteren Hilfskommittees erreichte vom britischen Home Secretary Sir Samuel Hoare 1938 die Zusage, dass alle Kinder bis 17 Jahre nach England kommen können sofern deren Unterhalt und die Kosten übernommen wurden. Auf diese Weise organisierte man bis 1939 die Ausreise von 9000 bis 10.000 Kinder; das Wiener Zentrum kümmert sich darum, die Kinder auszuwählen und ihren Transport zu organisieren, Darton, An Account of the Friends Committee for Refugees and Aliens, First Known as the Germany Emergency Committee of the Society of Friends, 1933-1950, $50-51$. 
viele bereits Episoden der Verfolgung und Inhaftierung erlebt hatten und die nun einem Krieg entgegensahen, der sie vor weitere schwere Konflikte stellen würde, zuletzt im Quäkerzentrum selbst, das von der Vielzahl von Menschen, die Zuflucht und Schutz suchten, förmlich erdrückt wurde. ${ }^{94}$

In Prag, wo er auf dem Höhepunkt der Sudetenkrise und rund eine Woche vor Abschluss des Münchner Abkommens eintraf, sah Pickett die Züge mit deutschen Sozialdemokraten und Tschechen, die in Erwartung einer deutschen Invasion aus dem Sudetenland in die Hauptstadt geflüchtet waren. Bei einem Treffen mit tschechischen Quäkern, zu dem auch Corder Catchpool aus England angereist war, war die desparate Lage greifbar: Die Anwesenden rechneten mit Krieg und Besatzung, die weitere Verfolgungswellen nach sich ziehen während sie gleichzeitig ein weiteres Schlupfloch für die Flüchtlinge aus Deutschland schließen würden. ${ }^{95}$

In Wien, das Pickett als „anxious place“ empfand, war er erschüttert von den Eindrücken der Verfolgungen sowie eines unter Hochdruck arbeitenden Flüchtlingszentrums in der Singerstraße. 400 Menschen drängten sich bei seinem Besuch vor dem Zentrum, „the one spot in Vienna, where non-Aryan Jews (sic!) can get any counsel and help in getting out of Germany“. Hier befanden sich acht Mitarbeiter in einem aussichtslosen Kampf gegen die Zeit und fehlende Ressourcen. Noch schwerer wog, dass diese Arbeit aufgrund der nach wie vor mangelnden Bereitschaft der Regierungen, den Verfolgten einen sicheren Hafen zu bieten, ins Leere zu laufen drohte. ${ }^{96}$

Unter dem Eindruck einer „overwhelming flood of dispossessed people who appeal to Friends for aid“ sickerte bei allen Beteiligten spätestens jetzt die Erkenntnis durch, dass alles Gewicht auf die Hilfe bei der Auswanderung gelegt werden musste. Dies betraf alle Ebenen - angefangen bei der Zentrale in Philadelphia, in der die Refugee Section nun personell aufgestockt wurde, über die Lobbyarbeit zugunsten der Lockerung der gesetzlichen Einreisebeschränkungen bis in die Quäkerzentren in Europa, wohin das AFSC nun weitere Freiwillige entsandte. ${ }^{97}$ Ein Refugee Committee, das gleichzeitig in Hertha Kraus' Haus in Bryn Mawr zusammenkam, widmete sich unterdes weiterhin der case work gegenüber denjenigen, denen die Flucht in die USA gelungen war. ${ }^{98}$

94 Miller, Witness for Humanity: A Biography of Clarence E. Pickett., 173-174.

95 Eintrag 23/9/1938, in: AFSCA, Clarence Pickett Journals 1920-1936

96 Eintrag 26/9/1938, in: AFSCA, Clarence Pickett Journals 1920 -1936.

97 Annual Report 1938, in: AFSC-Bulletin 137 (1939).

98 Auffällig an der Arbeit des Refugee Committee war, dass diese Arbeit im Kontrast zu den meisten anderen Abteilungen des AFSC nicht nur weiblich geführt war, sondern auch fast ausschließlich weibliche Mitglieder hatte. Permanent mit dem Komitee verbunden waren etwa Ve- 
Den letzten Anstoß gaben die Ereignisse des 9. und 10. November 1938, als im gesamten Deutschen Reich Synagogen brannten und die Nationalsozialisten eine neue, ungehemmte Welle der Gewalt gegen die jüdische Bevölkerung entfesselten. Unmittelbar nach den Pogromen stellte Pickett eine Anfrage bei der deutschen Botschaft, ob eine Hilfsaktion im Reich zugunsten der jüdischen Bevölkerung willkommen wäre. Bei einem Treffen in der Botschaft am darauffolgenden Tag erhielt das AFSC grünes Licht für ein begrenztes Programm. ${ }^{99}$ Noch am selben Tag versicherten sich Rufus Jones und Robert Yarnall bei einem Treffen im State Department der grundsätzlichen Zustimmung der amerikanischen Regierung für eine Hilfsaktion. ${ }^{100}$

Kurz darauf verfassten Rufus Jones und Clarence Pickett einen Brief, der an alle Quäkergemeinden im Land zur Verlesung nach dem sonntäglichen Gottesdienst versandt wurde: „Abundant evidence has come to the office of the American Friends Service Committee revealing a profound shock to our spiritual life by the Jewish and non-Aryan persecutions in Germany“, hieß es darin. Deutlich wurde, dass der Brief vor allem darauf abzielte, spontane öffentliche Proteste aus den Reihen der Quäker zu vermeiden, die die geplanten Aktionen eventuell gefährdet hätten. Verwiesen wurde vielmehr auf die bestehende Arbeit in Wien und im eigenen Land, verbunden mit der Aufforderung, sich auf die Immigration deutscher Flüchtlinge in der kommenden Zeit vorzubereiten. ${ }^{101}$

Am darauffolgenden Tag und damit eine knappe Woche nach der „Reichspogromnacht“, entschied das AFSC, eine Delegation nach Deutschland zu schicken, die vor Ort den Bedarf und die Bedingungen für eine Hilfsaktion ergründen sollte. Mittlerweile waren von Vertrauensleuten in ganz Deutschland Berichte zu

teraninnen der Kinderspeisungen wie Julia Branson, aber auch Anne Martin und Elizabeth Yarnall, Barbara Cary, Lydia Cadbury und Esther Thomas. Siehe die Sitzungsprotokolle des Refugee Committee 9/11/1938; 8/12/1938; 13/12/1938, in: AFSCA, General Files, Foreign Service, Country: Germany 1938 (Refugee Committee).

99 Der Geschäftsträger der Botschaft, Hans Dieckhoff, versicherte, dass das Regime dem keine Steine in den Weg legen würde, wenn die Aktionen auf die Ausreise der betroffenen Personen gerichtet wären. Treffen Rufus Jones / Bob Yarnell mit dem Geschäftsträger der Botschaft, Dr. Hans Dieckhoff, und Dr. Hans Thomsen in der dt. Botschaft 16/11/1938:, in: AFSCA, General Files, Foreign Service, Country: Germany 1938 (Delegation to Germany).

100 Robert Yarnall und Rufus Jones, Treffen mit Messerschmidt und Pierrepont Moffit (State Department) 16/11/1939, in: AFSCA, General Files, Foreign Service, Country: Germany 1938 (Delegation to Germany).

101 Das Schreiben bat in diesem Sinn vor allem um Spenden, um Bürgschaften und über die Bereitschaft zur vorübergehenden Aufnahme von Flüchtlingen nach der Ankunft im Land. Rundschreiben 17/11/1938, in: AFSCA, General Files, Foreign Service, Country: Germany 1938 (General). 
den Pogromen und Bestandsaufnahmen zum Bedarf an direkter Hilfeleistung eingeholt worden. ${ }^{102}$ Während sich die Gruppe von drei Quäkern - Rufus Jones, Robert Yarnall sowie ein weiteres Mitglied des AFSC-Executive Board, George Walton, Rektor einer Quäkerschule außerhalb von Philadelphia - am 2. Dezember an Bord der Queen Mary begab, führte das AFSC Gespräche mit Vertretern des JDC und anderer jüdischer Organisationen und bot an, deren Interessen in Deutschland wahrzunehmen. ${ }^{103}$

Der quasi-offizielle Charakter des Unternehmens wurde vor Abfahrt nochmals durch ein Gespräch Clarence Picketts mit Jay Pierrepont Moffat aus dem State Departement unterstrichen. Moffat versicherte Pickett, das State Department sei „,a hundred per cent for your delegation going“. Er bat das AFSC lediglich, sich mit Myron Taylor abzustimmen, der im Namen der Inter-Governmental Commission for German Refugees, die aus der Evian-Konferenz hervorgegangen war, ebenfalls Verhandlungen mit dem Regime führte, auch um zu vermeiden, dass die deutsche Regierung in der Lage wäre, „to drive a wedge between your delegation and the Inter-Governmental Committee“.104

Auch vor diesem Hintergrund war das AFSC bei der gesamten Aktion auf Geheimhaltung bedacht, um die Mission nicht zu gefährden - ohne Erfolg. Noch während sich die Delegation auf See befand, kündigten „Screaming headlines“ in der Presse von Philadelphia von der „heroic mission“ der Quäker zugunsten der deutschen Juden. Wie heikel dies aus Sicht des AFSC war, wird an der Reaktion Clarence Picketts deutlich, der zunächst das State Department davon unterrichtete, dass die Aufmerksamkeit gänzlich den Intentionen der Quäker widersprach, und gleichzeitig die deutsche Botschaft mit der Bitte kontaktierte, nach Möglichkeit Sorge dafür zu tragen, dass nichts davon in der deutschen Presse publik werde. Dieses Ziel wurde ebenfalls verfehlt - bei der Ankunft in Berlin empfing die Quäker ein Artikel Goebbels’ im Völkischen Beobachter, in dem er die Be-

102 Eine Vielzahl von Berichten mit dichten Beschreibungen der Ereignisse - verfasst von Gewährsleuten der Quäker etwa aus Bayern, Berlin, Frankfurt am Main und Ostpreußen, allesamt verfasst im Zeitraum von Mitte November bis Anfang Dezember - findet sich in: AFSCA, General Files, Foreign Service, Country: Germany 1938 (Delegation to Germany).

103 Mit Max Warburg besprach Clarence Pickett die Möglichkeit, jüdische Auswandererkolonien in Europa und Lateinamerika zu gründen. Treffen mit Joe Hyman, George Warren und Paul Baerwald (JDC) 23/11/1939 in: AFSCA, General Files, Foreign Service, Country: Germany 1938 (Delegation to Germany).

104 Moffat war weniger begeistert von der Idee, ein direktes Gespräch mit Hitler zu suchen, sicherte Pickett jedoch ansonsten mehrfach die Unterstützung des Unternehmens zu und gab dem AFSC Kontakte in Berlin zur US-Botschaft. Geheimes Gesprächsprotokoll Clarence Pickett über die Unterredung mit Pierrepont Moffat im State Department 25/11/1938, in: AFSCA, Foreign Service, Country: Germany 1938 (Delegation to Germany). 
mühungen der „Quäkersekte“ in ätzendem Ton lächerlich zu machen versuchte. ${ }^{105}$

Was folgte, war „an episode long on drama, if short on results“106 und je nach Sichtweise ein Beispiel unerschrockener Quäkerdiplomatie oder der fruchtlose und bestenfalls naive Versuch, den Nationalsozialisten tatsächliche Konzessionen abzuringen. ${ }^{107}$ Eine Konferenz mit Paul Sturge (FSC), Hans Albrecht sowie Howard Elkinton - einem Unternehmer aus Philadelphia, der seit Anfang des Jahres der Nachfolger Martins als Leiter des Berliner Zentrums war - machte eindringlich den Ernst der Lage klar. Man war sich einig, dass unter den gegenwärtigen Umständen die Organisation einer Massenauswanderung die einzige verbliebene Option war.

In einem Schreiben von Rufus Jones an den Vorsitzenden der Reichsvereinigung der Deutschen Juden spiegelte sich diese Erkenntnis wider: „Feeding does no longer seem to be the right line of service“, schrieb Jones und verkündete, die Quäker seien bereit, „to undertake the leadership of a world-wide movement to migrate the Jews in Germany to other countries“. Er zeigte sich zudem überzeugt, „that we can open doors for such a vast migration“, und verwies darauf, dass die Geschichte des AFSC als Beleg dienen möge für „our capacity to undertake a very large piece of work“.108

Gespräche mit Cora Berliner, Leo Baeck, Otto Hirsch und Paul Eppstein von der Reichsvereinigung ${ }^{109}$ verliefen entlang der gleichen Linie. Berliner berichtete von der verschärften Situation nach den Novemberpogromen und der vergeblichen Suche nach sicheren Häfen für die Flüchtlinge im Ausland. Die verzweifelte

105 Guy Aiken, „The American Friends Service Committee’s Mission to the Gestapo,“ Peace\&Change 42, 2 (2017), 214.

106 Schmitt, Quakers and Nazis: Inner Light in Outer Darkness, 107.

107 Die Reise der Quäkerdelegation ist gut dokumentiert durch die Teilnehmer, deren umfangreiche Notizen ein praktisch lückenloses Bild der Ereignisse ergeben. Ein lückenloser Bericht findet sich bei Aiken, „The American Friends Service Committee's Mission to the Gestapo.“; Schmitt, Quakers and Nazis: Inner Light in Outer Darkness, 107-109. Rufus Jones und George Walton haben ihre Erinnerungen in längeren Artikeln veröffentlicht. Rufus Jones, „Our Day in the German Gestapo“, in: The American Friend, 10/7/1947; George Walton, „Two Weeks in Berlin December 1938“, in: The Georgian, George School June 1939, Vol X, Number 6.

108 Rufus Jones an den Reichsvertreter der Deutschen Juden 10/12/1938, in: AFSCA, General Files, Foreign Service, Country: Germany 1938 (Delegation to Germany).

109 Kurze Zeit zuvor war die Reichsvereinigung zwangsweise in „Reichsvereinigung der Juden in Deutschland“ umbenannt worden. Die Delegation traf darüber hinaus auch andere Vertreter der deutschen Juden wie Wilfrid Israel, einen der entscheidenden Organisatoren der Kindertransporte. Einen dauerhaften Eindruck hinterließ auch der Besuch der Ausstellung „Der ewige Jude“. Reisenotizen Robert Yarnall 09/12/1939; 12/12/1939 in: AFSCA, General Files, Foreign Service, Country: Germany 1938 (Delegation to Germany). 
Lage - „Fear! Fear!“ notierte Robert Yarnall über die Atmosphäre des Treffens wurde greifbar, als Baeck und Berliner die Einrichtung von Übergangskonzentrationslagern für jüdische Flüchtlinge in den USA anregten, in denen diese bis zur Ausschöpfung der jährlichen Quotenregelungen verbleiben könnten. ${ }^{110}$

Für die Quäkerdelegation ging es nun vor allem darum, eine möglichst hochrangige Regierungsstelle ausfindig zu machen, die bereit war, die Delegation zu empfangen und über eine eventuelle, von den Quäkern organisierte, Ausreise der Verfolgten zu verhandeln. Gespräche mit dem Leiter des Winterhilfswerks der Nationalsozialistischen Volkswohlfahrt (NSV), Erich Hilgenfeldt, verliefen ergebnislos. Bei einem Treffen mit dem amerikanischen Generalkonsul Raymond Geist wurden weitere Möglichkeiten besprochen. Ein direktes Gespräch mit Hitler wurde als aussichtslos verworfen und Geist regte an, den Weg über die Gestapo zu gehen. Ein Treffen mit Vertretern des deutschen Auswärtigen Amtes brachte den Durchbruch und ein Treffen im Reichssicherheitshauptamt, dem Sitz der Gestapozentrale, wurde für die kommende Woche anberaumt. ${ }^{111}$ In der Zwischenzeit reiste Rufus Jones nach London, um mit dem FSC und Myron Taylor das weitere Vorgehen zu koordinieren. Letzterer war jedoch, wie sich zeigte, nicht begeistert von der Paralleldiplomatie der Quäker. ${ }^{112}$ Yarnall fuhr unterdessen nach Wien, um den dortigen Bedarf zu eruieren. ${ }^{113}$

110 Berliner wurde zitiert mit: „At present (...) relief not pressing, but of first importance is large scale emigration. Suggests we set up in America huge concentration camp where we can hold those who qualify until they can come in under 27.000 per year quota." Reisenotizen Robert Yarnall, Gespräch mit Vertretern der Reichsvertretung der Deutschen Juden 10/12/1939; 20/12/1939 in: AFSCA, General Files, Foreign Service, Country: Germany 1938 (Delegation to Germany). Cora Berliner wurde 1942 deportiert und vermutlich bei Maly Trostenez ermordet. Otto Hirsch kam 1941 im Lager Mauthausen um; Paul Eppstein starb in Theresienstadt 1944; Leo Baeck überlebte das Lager Theresienstadt und starb 1956 in London.

111 Die Vertreter des Auswärtigen Amtes gaben den Quäkern gegenüber ungeschminkt zu verstehen, dass alle Politik darauf gerichtet war, so viele Juden wie möglich zur Ausreise zu bewegen. Jede Initiative, die dieses Ziel unterstütze, sei dabei willkommen. Reisenotizen Robert Yarnall 15/ 12/1939, in: AFSCA, General Files, Foreign Service, Country: Germany 1938 (Delegation to Germany).

112 Aiken, „The American Friends Service Committee’s Mission to the Gestapo.“, 215.

113 Robert Yarnall vermerkte, dass Jones bei einem Treffen mit Taylor und einem Vertreter des US State Department bestätigt wurde, dass sich das AFSC ,in coordination with their plans“ befinde. Reisenotizen Robert Yarnall 14/12/1939, in: AFSCA, Foreign Service, Country: Germany 1938 (Delegation to Germany). 
Am 19. Dezember schließlich kam es zu einem Gespräch mit zwei Mitarbeitern von Gestapochef Reinhard Heydrich, ${ }^{114}$ in dem die drei Männer Freundschaftsbekundungen gegenüber Deutschland und den Verweis auf die humanitäre Hilfe nach dem Ersten Weltkrieg mit dem Angebot verbanden, Hilfe für die deutschen Juden bei ihrer Ausreise aus Deutschland zu leisten. ${ }^{115}$ In einem vorbereiteten Schreiben für die deutschen Gesprächspartner hieß es:

We represent no government, no international organizations, no parties, no sects (...). We have come now in the same spirit as in the past and we believe that all Germans who remember the past and who are familiar with our ways and methods and spirit will know that we do not come to judge or to criticize or to push ourselves in, but to inquire in the most friendly manner whether there is anything we can do to promote life and human welfare and to relieve suffering.

Laut Yarnalls Bericht erhielten die Quäker nach kurzer Unterredung tatsächlich die Zusicherung von Heydrichs Vertretern, die „Evakuierung“ der deutschen Juden unterstützen und sich zu diesem Zweck frei in Deutschland und Österreich bewegen zu können. Eine schriftliche Bestätigung seitens der Gestapo gab es dafür aber nicht. ${ }^{116}$

Was folgte, war ein Gespräch mit Reichsbankpräsident Hjalmar Schacht, der zeitgleich auch mit der Inter-Governmental Commission und deren Vertreter George Rublee über die finanzielle Seite jüdischer Emigration verhandelte. Schacht versicherte den Quäkern, dass er ihre Initiative unterstütze, auch wenn er anmerkte, die Frage sei „too big for charity“. Am Ende des Gesprächs gab auch er der Delegation zu verstehen, dass, was auch immer die Planungen seien, die Zeit dränge, denn, so Schacht unheilschwanger ,nobody knows what will happen in this country tomorrow“. ${ }^{117}$

114 Die Namen werden mit Standartenführer Dr. Erlinger und Regierungsrat Lischka angegeben, in: Report of Quaker Delegation 19/12/1938, in: AFSCA, General Files, Foreign Service, Country: Germany 1938 (Delegation to Germany).

115 Auch auf die langjährige Gegnerschaft gegenüber dem Versailler Vertrag wurde verwiesen ebenso wie den Kampf gegen die Blockade und auf die Kinderspeisungen in Deutschland und Österreich. Statement 20/12/1939, in: AFSCA, General Files, Foreign Service, Country: Germany 1938 (Delegation to Germany).

116 Statement 20/12/1939, in: AFSCA, General Files, Foreign Service, Country: Germany 1938 (Delegation to Germany).

117 Gespräch mit Hjalmar Schacht, in Reisenotizen Robert Yarnall 20/12/1938, in: AFSCA, General Files, Foreign Service, Country: Germany 1938 (Delegation to Germany). Nach den Notizen George Waltons versprach Schacht auch, Hitler und Göring die Pläne der Quäker vorzulegen (es gibt keine Anzeichen dafür, dass dies jemals geschah). George Waltons Eindruck von Schacht war insgesamt positiv: „A man of big heart and mind - one of the great men of his generation- who 
Trotz der Unsicherheit und des Mangels an direkten Resultaten, wurde die Mission vom AFSC als Erfolg gewertet. Sie habe, wie Pickett aus Rufus Jones' „remarkable report“ schloss, den deutschen Juden eine Botschaft des guten Willens von amerikanischen Christen überbracht und ihnen gezeigt, dass sie nicht ohne Freunde in der Welt seien. ${ }^{118}$ Nun ging es vor allem darum, die Aufmerksamkeit für die Mission nach der Rückkehr Anfang Januar 1939 zu nutzen und unmittelbare Taten folgen zu lassen. Ungeachtet der Tatsache, dass „rapid emigration is the imperative need at present“, hielt der Bericht es aus praktischen und strategischen Gründen für angezeigt, parallel auch ein Hilfsprogramm in Deutschland zu starten: „(P)lans should be made at once“, hiess es in diesen Sinn, „to assist with some relief as well, partly for the direct good resulting to those who suffer and who are not now adequately provided for and partly for the toe hold such work will give for other help“. Angeregt wurde im Einzelnen eine Spendenkampagne und die Ernennung einer kleinen Gruppe von Quäkern, „to be sent over at once to supervise distribution of relief money and to assist and keep actively behind the rapid emigration program“. Auch die internationalen Bemühungen, insbesondere die Zusammenarbeit mit der Inter-Governmental Commission und den Vertretern des JDC in Europa sollten ausgebaut werden. ${ }^{119}$

Die beachtliche Aufmerksamkeit, welche die Rückkehr der Delegation in der Presse hervorrief, wollte das AFSC nutzen, um sich in den USA - nach dem Vorbild der britischen Quäker - als zentrale Stelle für die Organisation der Auswanderung aus Deutschland in Position zu bringen. Bereits am 4. Januar beschloss das AFSC, eine Kommission $\mathrm{zu}$ berufen, die in den folgenden Monaten erneut nach Deutschland reisen und die Bedingungen für eine größere Evakuierungsaktion aus Deutschland schaffen sollte. Hierfür erhielt das AFSC im unmittelbaren Anschluss 100.000 US-Dollar vom Jewish Joint Distribution Committee. Herbert Hoover sicherte parallel zu, eine halbe Million Dollar zur Verfügung $\mathrm{zu}$ stellen. ${ }^{120}$

regardless of pride, holds on to do a hard job“. Notizen George Walton 20/12/1939, in: AFSCA, General Files, Foreign Service, Country: Germany 1938 (Delegation to Germany).

118 In Jones' Bericht hieß es dazu: „It can be said, I think, that in the main we did what we went to do. We carried by our presence at this crisis in their lives a silent message of good will and we made those, who needed help most, understand that there was someone who cared and who sympathized and who suffered with them, and who was eager to help. From that point of view the journey was worth making and it reached its goal. We felt and found each other." Bericht Rufus Jones zur Reise nach Deutschland 6/1/1939, in: AFSCA, General Files, Foreign Service, Country: Germany 1939 (Delegation to Germany cont.).

119 Recommendations of the German delegation 4/1/1939, in: AFSCA, Foreign Service, Country: Germany 1939 (Delegation to Germany cont.).

120 Auch Ex-Präsident Herbert Hoover trat an Pickett mit der Versicherung heran, im Erfolgsfall persönlich Sorge dafür zu tragen, „to find sufficient funds to do such feeding as was needed in 
Gleichzeitig begann das AFSC Gespräche mit Regierungsstellen. „Emigration on this scale“ (man rechnete mit ca. 50.000 Menschen pro Jahr, DM) sei „too large a job for private charity“, hieß es hierzu in den Empfehlungen des AFSC.Vielmehr handele es sich um einen ,government job“. ${ }^{121}$ Mit Arbeitsministerin Frances Perkins erörterte Pickett die Aussichten für eine Erhöhung der Quoten für unbegleitete Kinder, denen (mit Blick auf die laufenden „Kindertransporte“) ein Hauptaugenmerk galt. Mit dem State Department und dem Justizministerium eruierte das AFSC darüber hinaus, an die Gespräche mit den Vertretern der Reichsvereinigung anschließend, die Einrichtung von Transitlagern für jüdische Flüchtlinge in den USA. ${ }^{122}$

Die Ernüchterung folgte prompt und umfassend. Im April 1939 verweigerte der Kongress einem Gesetzesentwurf die Zustimmung (der nach ihren Hauptsponsoren benannten Wagner-Rogers Bill), der zunächst 20.000 Kindern die Einreise in die USA erlaubt hätte. Im Vorfeld hatte das AFSC große Hoffnungen in diesen Entwurf gesetzt und wenngleich Clarence Pickett mit dem Widerstand eines „strong reactionary bloc in Congress“ gerechnet hatte, war dieses Ergebnis ein Schock. ${ }^{123}$ Eine Mehrheit sah dieses Gesetz jedoch offenbar als Einfallstor für eine Erhöhung der Zuwanderungsquoten. Für Pickett, der mit Yarnall und weiteren Quäkern bei den öffentlichen Anhörungen für den Gesetzesentwurf als Zeuge aufgetreten war, war dies eine schmerzliche Enttäuschung. Damit war jede Hoffnung auf einen Neuanfang im Keim erstickt.

Umsonst waren auch alle Hoffnungen, die USA würden international als Vorreiter einer liberaleren Haltung in der Frage der Einwanderung deutscher Flüchtlinge dienen. In einem Bericht, den Robert Balderston, einer der Quäkergesandten, die das AFSC nach Deutschland geschickt hatte, der Refugee Section des AFSC erstattet hatte, war dieser Gedanke prominent gewesen. Balderston sah eine neue Quotenregelung als „very effective wedge“ im Umgang mit anderen

Germany“. Es wurde beschlossen James Vail, Robert Balderston und Harvey Perry als Commissioner zu schicken. Memorandum Anfang 1939 (n.n.), in: AFSCA, Foreign Service, Country: Germany 1939 (Delegation to Germany cont.).

121 Recommendations of the German delegation 4/1/1939, in: AFSCA, General Files, Foreign Service, Country: Germany 1939 (Delegation to Germany cont.)

122 Gespräche mit Mr. Frank Ritchie (ACCGR) und mit President's committee on German refugees. Gepräch am 10/1/1939 mit George Messersmith (SD) zu Transitcamps und mit Arbeitsministerin Frances Perkins besprochen zu Kinderquoten. 20/1: Gespräch mit Solicitor General Robert Jackson wird wegen Transitcamps angesprochen. Dieser liess jedoch durchblicken, dass das State Department nicht begeistert sei, in: Memorandum Anfang 1939 (n.n.), in: AFSCA, Foreign Service, Country: Germany 1939 (Delegation to Germany cont.)

123 Refugee Committee Minutes 9/2/1939, in: AFSCA, AFSC Minutes 1939. 
Regierungen, vor allem in Europa, die insbesondere auf ein Zeichen aus den USA warteten. ${ }^{124}$

Auch mit Blick auf die Unterstützung der Regierung stießen die Bemühungen schnell an ihre Grenzen. Bereits bei ersten Gesprächen im State Department nach der Rückkehr der Delegation aus Deutschland hatte sich gezeigt, dass die Aktivitäten der Quäker immer im Zusammenhang mit den Fortschritten der InterGovernmental Commission gesehen wurden und aus Sicht der Außenpolitik wenig eigenes Gewicht hatten. ${ }^{125}$ Als auch diese Bemühungen zunehmend im Sand verliefen, verlor sich auch das ohnedies geringe Interesse an der AFSC-Aktion. Auch Clarence Picketts persönliche politische Verbindungen gelangten hier rasch an ihre Grenzen. Im Januar stellte er den Plan des AFSC bei einem Treffen mit Eleanor Roosevelt vor. Am Ende erwiesen sich jedoch die Bedenken des State Departments auch hier als zu stark. Nach einem ,extremely unpleasant and unsatisfactory interview“ mit dem stellvertretenden Außenminister George Messersmith musste das AFSC erkennen, dass es auf keine weitergehende Unterstützung zählen konnte. Aus Picketts Sicht war dafür nicht zuletzt der Einfluss von Außenminister Cordell Hull ausschlaggebend, den er nach Picketts Biografen verantwortlich machte „for the lack of decisive action by the United States in rescuing as many Jews as possible“. ${ }^{126}$ Hull war dabei gleichwohl nur einer von vielen, die sich in dieser Frage in der aufgeheizten, von anti-semitischen und xenophoben Stimmen geprägten Debatte um Einwanderung zögerlich gegenüber einer Aufnahme jüdischer Flüchtlinge verhielten. ${ }^{127}$

Wie widrig dieses Klima war, lässt sich leicht aus einer Broschüre schließen, die das AFSC gemeinsam mit einer Reihe vorwiegend jüdischer und christlicher Flüchtlingshilfskomitees im Frühjahr 1939 unter dem Titel Refugee Facts veröffentlichte: Die Schrift richtete sich an die amerikanische Öffentlichkeit mit einem

124 Balderston vertrat vehement die Idee der Transitlager, weil er das Hauptproblem darin sah, dass die Länder, die Grenzen mit Deutschland teilten, vor allem von der Angst getrieben wären, die Bereitschaft als sicherer Hafen zu fungieren, sei gleichbedeutend mit der endgültigen Aufnahme der Flüchtlinge. Bericht Robert Balderston an Refugee Section 28/4/1939, in: AFSCA, AFSC Minutes 1939.

125 Conference at Washington with Robert Yarnall, James Vail and Homer Morris mit Pierrepont Moffat und Illig, State Department 19/1/1939, in: Recommendations of the German delegation 4/1/ 1939, in: AFSCA, Foreign Service, Country: Germany 1939 (Delegation to Germany cont.)

126 Pickett zitiert nach Miller, Witness for Humanity: A Biography of Clarence E. Pickett, 187; $190-191$.

127 Siehe zum breiteren Kontext Richard Breitman and Allan J. Lichtman, FDR and the Jews (Cambridge, Massachusetts: The Belknap Press of Harvard University Press, 2013); Henry L. Feingold, The Politics of Rescue; the Roosevelt Administration and the Holocaust, 1938-1945 (New Brunswick, N.J.,: Rutgers University Press, 1970). 
Appell, die Aufnahme von Flüchtlingen aus Deutschland in erster Linie als „opportunity for enriching American life both commercially and culturally“ zu sehen. Sie betonte den im Durchschnitt hohen Bildungsgrad der deutschen Flüchtlinge und unterstrich insbesondere den ökonomischen Nutzen, den diese Menschen, als Unternehmer, Fachkräfte, aber auch als Konsumenten bringen würden. Sie hob hervor, dass viele der Aufnahmesuchenden Intellektuelle seien, die helfen würden „to make our country the cultural centre of the modern world by their presence here“. Systematisch versuchte die Broschüre damit dem Vorurteil zu begegnen, die Flüchtlinge stellten eine Gefahr für die Arbeitsplätze amerikanischer Arbeiter dar. Daneben trat noch ein weiteres Motiv in den Vordergrund: Die Broschüre verwendete viel Raum darauf nachzuweisen, dass entgegen der allgemeinen Vorstellung keineswegs alle Flüchtlinge Juden seien, sondern vielmehr die Gruppe der Hilfesuchenden neben politischen Gegnern des Regimes auch aus einer großen Zahl von Christen bestehe, die erst durch die Rassengesetze der Nationalsozialisten als solche definiert worden seien. Hier wurde vor allem anderen deutlich, dass in den Vorbehalten gegen die Aufnahme von Flüchtlingen ein gehöriger Teil anti-semitischer Ressentiments mitschwang. Die „Refugee facts“ waren so wichtig, so machte die Schrift deutlich, weil es nur natürlich sei, ,that many people who are uninformed, should be easy prey for anyone who wishes to increase anti-alienism or anti-semitism in our land. Every American is now aware that there are many such organisations in our country which are exerting themselves mostly in furtive ways to increase racial feeling among us. The truth and intelligent assistance to the newcomers together will dispel these fears. " ${ }^{128}$ Ressentiments und Nativismus, vor allem aber die Furcht der Politiker, sich diesen Strömungen in der Frage der Einwanderung entgegenzustellen, waren starke und oftmals übermächtige Gegner, denen sich die Befürworter der Aufnahme deutscher Flüchtlinge gegenüber sahen.

128 Die Liste der mitunterzeichnenden Organisationen zeigt die Breite des Bündnisses an, als dessen Teil die Quäker agierten: Mitglieder neben den Quäkern waren das American Committee for Christian German Refugees, das American Jewish Committee, der American Jewish Congress, das American Jewish Joint Distribution Committee, das Committee for Catholic Refugees from Germany, der Council of Jewish Federations and Welfare Funds, das Emergency Committee in Aid of Displaced Foreign Medical Scientists, das Emergency Committee in Aid of Displaced Foreign Scholars, der Federal Council of Churches of Christ in America, die German Jewish Children's Aid, die Hebrew Sheltering and Immigrant Aid Society, das Intercollegiate Committee for Student Refugees, der International Migration Service, der International Student Service, die Jewish Agricultural Society of America, der Musicians Emergency Fund, das National Board des Young Men's and Young Women's Christian Associations, der National Council of Jewish Women und die Zionist Organisation of America. Refugee Facts, in: AFSC-Bulletin 132 (1939). 
In den verbleibenden Monaten vor Kriegsausbruch setzte das AFSC seine Arbeit ungeachtet aller Rückschläge fort. Es betrieb weiter Lobbyarbeit für eine Aufnahme von Flüchtlingen aus Deutschland und leistete praktische Hilfe für die Wenigen, die in dieser Zeit allen Widrigkeiten zum Trotz die USA erreichten. Auf Initiative von young friends aus dem Mittleren Westen eröffnete im Sommer 1939 das „Scattergood Hostel“ in West Branch, Iowa, seine Türen ,as a centre at which newcomers could recuperate from the strain of living in Central Europe and learn to adjust themselves to American ways of Life“. ${ }^{129}$ Hertha Kraus intensivierte indes die Bemühungen um Zufluchtsorte für deutsche Flüchtlinge außerhalb der USA. Sie unternahm zu diesem Zweck persönlich Reisen nach Kuba und versuchte über diplomatische Kanäle neue Möglichkeiten in mehreren mittel- und lateinamerikanischen Ländern $\mathrm{zu}$ eröffnen. ${ }^{130}$

In Deutschland blieb dem AFSC zur selben Zeit nicht mehr übrig, als die Arbeit der europäischen Zentren weiter nach Kräften zu unterstützen. Die Gruppe aus fünf Quäkern, welche das AFSC dann tatsächlich im Frühjahr 1939 entsandte, erfüllte im Wesentlichen diesen Zweck. ${ }^{131}$ Der Plan, die Zentren mit jungen Quäker-Freiwilligen zu füllen, scheiterte dagegen frühzeitig am Einspruch des State Department, das die Aktion auf wenige Personen begrenzt wissen wollte. ${ }^{132}$ Aus den Berichten von James Vail und Robert Balderston geht hervor, dass die Arbeit sich unter dem Druck der Ereignisse frühzeitig von klassischer relief work hin zu Ausreisefragen verlagerte. Vail arbeitete $\mathrm{zu}$ diesem Zweck eng mit der InterGovernmental Commission unter ihrem neuen Leiter Sir Herbert Emerson sowie insbesondere mit Morris Troper, dem europäischen Direktor des JDC in Paris, in einer „fellowship of suffering“ zusammen. ${ }^{133}$ Nach der Annexion der Tschechoslowakei durch Deutschland im April 1939, die eine neue Welle der Flucht auslöste, kooperierten die Quäker mit dem JDC und anderen Hilfsorganisationen ebenso angesichts der Tragödie um die St. Louis, ein Flüchtlingsschiff, dem mit

129 Seit Ende 1938 hatte sich ein eigenes Scattergood Committee im AFSC unter dem Vorsitz von Albert und Anne Martin mit der Einrichtung des Zentrums beschäftigt. Zur Geschichte des Hostels siehe Luick-Thrams, Out of Hitler's Reach: The Scattergood Hostel for European Refugees 19391943.

130 Minutes Refugee Section 26/4/1939, in: AFSCA, AFSC Minutes 1939.

131 James Vail, der nach Berlin ging, sowie die Ehepaare Harvey und Juliana Perry, die in Wien ihren Hauptsitz aufschlugen, sowie Martha und Robert Balderston, die von Frankfurt am Main aus operierten, waren allesamt während der 1920er Jahre in Deutschland tätig gewesen waren, Schmitt, Quakers and Nazis: Inner Light in Outer Darkness, 111.

132 Eleanor Slate (AFSC) an Robert Balderston 16/3/1939, in: AFSCA, General Files, Foreign Service, Country: Germany 1939 (European Commissioners).

133 Aufschlussreich ist in dieser Hinsicht das Memorandum von James Vail für Morris Troper 24/ 3/1939, in: AFSCA, General Files, Foreign Service, Country: Germany 1939 (James Vail). 
rund tausend deutschen Juden die Landung im sicheren Hafen von Havanna verweigert worden war und das im Juni 1939 nach Europa zurückkehren musste. In beiden Fällen und bei einer Reihe weiterer Gelegenheiten beteiligten sich die Quäker an der Suche nach aufnahmebereiten Regierungen und nutzten ihre Kontakte etwa in die Niederlande und die skandinavischen Staaten. ${ }^{134}$ Darüber hinaus zählten die AFSC-Vertreter die Vermittlung eines Devisenabkommens für jüdische Auswanderer aus Wien zu ihren Erfolgen sowie den Aufbau einer landwirtschaftlichen Schule für junge Flüchtlinge in den Niederlanden (am Ort der Quäkerschule in Eerde/Ommen). Gerade letztere Aktivität zeigt einen bedeutenden Aspekt der Hilfe, die gerade im Licht der hohen Hürden, die der Einwanderung in die USA und anderswohin entgegenstanden, gezwungen war, harte Prioritäten zu setzen. ${ }^{135}$ In seinem Memorandum für das JDC erläuterte Vail die Erwägungen, die hinter der Landwirtschaftsschule in Eerde standen: Es sei größtes Gewicht darauf zu legen, dass die Gruppe der Flüchtlinge, die es unter den restriktiven Bedingungen in die USA schafften, sich tatsächlich als nützlich erweise, da alle anderen, auf die dies nicht zutraf, ,are certain to close the doors against those who are able to be serviceable““. ${ }^{136}$ Ein Gefühl der Begrenztheit der eigenen Möglichkeiten war vorherrschend. Im Rückblick auf die ersten Monate bilanzierte Balderston ganz in diesem Sinn, „that our greatest service has been that of cooperation“. ${ }^{137}$

Als Homer und Edna Morris sich Ende August als neue Quäkeremissäre auf die Reise nach Deutschland machten, waren die Schatten des drohenden Krieges schon allgegenwärtig. Bereits eine Woche vor ihrer Ankunft in England am 30. August war die Leitung des Berliner Zentrums an die deutschen und ameri-

134 Report of the European Commissioners 1/7/1939, in: AFSCA, General Files, Foreign Service, Country: Germany 1939 (European Commissioners).

135 Die amerikanischen Quäker Harvey und Juliana Perry errichteten im Auftrag des AFSC hierzu ein currency exchange system ein, durch das die Restriktionen umgangen werden sollten, die die Nazis vor der Ausfuhr jüdischen Besitzes aufbauteten. Auch dies kam 1939 zum Erliegen.

136 „In frankness, it must be said that there is substantial group of refugees who, perhaps through no fault of their own, will not, for one reason or another ever become constructive citizens in a new home. A useful figure of comparison is that of the contagious disease which must be dealt with at the point of origin. If undesirable immigrants are allowed, without adequate arrangements to enter new countries, they are certain to close the doors against those who are able to be serviceable. For them some tolerable life within Germany should be insisted upon as a condition of cooperation of other governments in complying with the fundamentally unreasonable demands of mass immigration. " Memorandum von James Vail für Morris Troper 24/3/1939, in: AFSCA, General Files, Foreign Service, Country: Germany 1939 (James Vail).

137 Report of the European Commissioners 1/7/1939, in: AFSCA, General Files, Foreign Service, Country: Germany 1939 (European Commissioners). 
kanischen Quäker übertragen worden - das englische Personal dagegen hatte Deutschland angesichts der Kriegsgefahr verlassen. Als das Ehepaar Morris schließlich im September in Berlin eintraf, waren alle Hoffnungen, in Deutschland selbst oder bei der Organisation der Ausreise deutscher Juden Hilfe leisten zu können, verflogen. Wenngleich das Zentrum vorerst geöffnet blieb, war schon aus Rücksicht auf die deutschen Quäker kein Spielraum mehr vorhanden. Edna Morris vermerkte in ihrem Tagebuch, die Quäker „maybe able to pull through the storm“, jedoch nur dann, wenn sie ihre Arbeit leise und „on unprovocative fields“ fortsetzten. ${ }^{138}$

In der Tat verblieb dem AFSC noch ein gutes Jahr, bevor das Zentrum seine Türen endgültig schloss, und es sollte bis 1945 dauern, bis die britischen und amerikanischen Quäker ins besiegte und zerstörte Deutschland zurückkehrten. Ein Großteil der Arbeit, die die Quäker zwischen 1933 und 1939 für die Opfer der nationalsozialistischen Verfolgungen geleistet haben, ist in Vergessenheit geraten, wobei die vollständige Diskreditierung der „pazifistischen Diplomatie“ nach 1938 eine bedeutende Rolle gespielt haben mag. Der Quäkeransatz, mit „beiden Seiten des Konflikts“, Opfern und Tätern ohne Vorbedingungen im Gespräch zu bleiben, war in Deutschland fraglos an seine Grenzen gestoßen. Dennoch war die Erfahrung der Hilfe in Deutschland in mehrfacher Hinsicht wegweisend. Insbesondere hatte sie das AFSC, wenn auch indirekt, zum Teil der internationalen diplomatischen Bemühungen der amerikanischen Außenpolitik werden lassen. Dies war ein wichtiger Schritt, umso mehr da sich die deutsche Aktion ab 1936 vor dem Hintergrund des Spanischen Bürgerkriegs mit einem weiteren Hilfseinsatz überschnitt, durch den das AFSC Gelegenheit erhielt, seine Identität und sein Renommee als eine gleichzeitig US-amerikanische und neutrale humanitäre Organisation zu stärken.

138 Tagebucheintrag Edna Morris 16/9/1939, in: AFSCA, Foreign Service: Germany 1939 (Diaries, Letters and Memoranda of Homer L. Morris and Edna W. Morris as Commissioners to Germany for the American Friends Service Committee 1939). 